\title{
Visual Stimulation Decorrelates Neuronal Activity
}

\author{
Mike W. Oram \\ School of Psychology, University of St. Andrews, St. Andrews, Fife, United Kingdom
}

Submitted 5 August 2009; accepted in final form 19 September 2010

\begin{abstract}
Oram MW. Visual stimulation decorrelates neuronal activity. $J$ Neurophysiol 105: 942-957, 2011. First published September 22, 2010; doi:10.1152/jn.00711.2009. The accuracy of neuronal encoding depends on the response statistics of individual neurons and the correlation of the activity between different neurons. Here, the dynamics of the neuronal response statistics in the anterior superior temporal sulcus of the macaque monkey is described. A transient reduction in the normalized trial-by-trial variability and decorrelation of the responses with both the activity of other neurons and previous activity of the same neuron are found at response onset. The variability of neuronal activity and its correlation structure return to the levels observed in the resting state 50-100 ms after response onset, except for marked increases in the signal correlation between neurons. The transient changes in the response statistics are seen even if there is little or no stimulus-elicited activity, indicating the effect is due to network properties rather than to activity changes per se. Modeling also indicates that the observed variations in response variability and correlation structure of the neuronal activity over time cannot be attributed to changes in firing rate. However, a reset of the underlying spike-generating process, possibly due to the driving input changing from recurrent to feedforward inputs, captures most of the observed changes. The nonstationarity indicated by the changes in correlation structure around response onset increases coding efficiency: compared with the mutual information calculated without regard to the transitory changes, the decorrelation increases the information conveyed by the initial response of modeled neuronal pairs by $\leq 24 \%$ and suggests that an integration time of as little as $50 \mathrm{~ms}$ is sufficient to extract $95 \%$ the available information during the initial response period.
\end{abstract}

\section{N T R O D U C T I O N}

The ability of the brain to encode information is determined by the response characteristics of the individual neurons and the correlation structure between the responses of different neurons. Reports of the neuronal coding of visual stimuli have typically analyzed data using sample windows that are fixed in both time and size (e.g., a window size of $250 \mathrm{~ms}$ starting 50 ms after stimulus onset). More recently, studies have started to examine changes in the response characteristics at different time points within the stimulus-elicited response (e.g., Amarasingham et al. 2006; Churchland et al. 2006; Muller et al. 2001; Smith and Kohn 2008). The study of the dynamics of response statistics can help elucidate the properties of underlying neural circuits and constrain computational models in addition to quantifying how dynamic changes in response characteristics influence the stimulus-related information carried by the responses.

To determine the impact of changes in response statistics over time in terms of information, it is necessary to measure response variability. For individual neurons, the more variable

Address for reprint requests and other correspondence: M. W. Oram, School of Psychology, University of St. Andrews, St. Andrews, Fife, KY16 9JU, the responses to a given stimulus, the less information those neurons can encode. The trial-by-trial variability increases from retina to the lateral geniculate nucleus (LGN) to primary visual cortex (V1) (Kara et al. 2000) and differences have been noted between cortical areas (e.g., visually responsive neurons in middle temporal and middle superior temporal [MT/MST] areas show greater trial-by-trial variability than motor-related neuronal activity in area 5; Maimon and Assad 2009). Studies frequently report the Fano factor (variance/mean spike count in a given sample window). Although some studies report Fano AQ: 1 factors <1 (Amarasingham et al. 2006; Bair 1999; Gur and Snodderly 2006; Gur et al. 1997; Kara et al. 2000) or $>2$ (Vogels and Orban 1991), Fano factors are typically $>1$ and $<1.5$ in LGN (Levine and Troy 1986; Oram et al. 1999; Reich et al. 1997), V1 (Bradley et al. 1987; Carandini 2004; Dean 1981; Geisler and Albrecht 1997; Tolhurst et al. 1981, 1983; Victor and Purpura 1996), V4 (McAdams and Maunsell 1999), inferotemporal cortex and anterior superior temporal sulcus (IT/STSa) (Amarasingham et al. 2006; Baddeley et al. 1997; Oram et al. 2002; Vogels et al. 1989; Wiener et al. 2001), MT (Britten et al. 1993; Buracas et al. 1998; Snowden et al. 1992; Uka and DeAngelis 2003), parietal and motor cortices (Lee et al. 1998; Maynard et al. 1999; Oram et al. 2001), and supplementary motor cortex (Averbeck and Lee 2003). Although the variability of neuronal responses has typically been examined using a "fixed" analysis window, the variability of the initial response is less than that in the sustained or latter part of stimulus- or motor-induced activity in premotor cortex, V1, IT, and STSa (Amarasingham et al. 2006; Churchland et al. 2006; Muller et al. 2001; Oram and Perrett 1992; Vogels and Orban 1991), suggesting that the initial transient response will be more informative than later parts of the response (Gershon et al. 1998).

If the responses of a neuron to repeated presentations of a stimulus are not Poisson distributed, as indicated when the Fano factor differs from unity, then the responses will have correlation over time (Kass and Ventura 2006; Oram et al. 1999, 2001). The correlation between successive time windows within the responses of a single neuron (sequential autocorrelation) has been found in parietal, MT, IT, and motor cortices (Bair et al. 2001; Lee et al. 1998; Oram et al. 2007; Osborne et al. 2004; Uka and DeAngelis 2003). Knowledge of both the time course of and how sequential autocorrelation varies with temporal window size provides insight into the temporal resolution of integration of signals available within the brain.

Evaluation of stimulus-related information coded by a population of neurons requires quantification of the correlations between the responses of different neurons when a stimulus is presented. Responses from simultaneously recorded neurons allow evaluation of both the correlation of the signal between neurons (estimated from the mean responses to different stim- AQ: A 
uli) and the between-neuron correlation of the trial-by-trial variability to different stimuli (the so-called noise correlation). The noise correlation between neurons may be small, particularly when receptive field similarity or overlap is small (Averbeck and Lee 2006; Bair et al. 2001; DeAngelis et al. 1999; Lee et al. 1998; Maynard et al. 1999; Smith and Kohn 2008). The influence of the between-neuron signal and "noise" correlation on encoded information is complex. Although the presence of correlation will reduce the entropy of the population response, the levels of correlation observed between cortical neuronal responses, $r=0.1-0.3$ (Bair et al. 2001; Gawne and Richmond 1993; Gawne et al. 1996; Kohn and Smith 2005; Lee et al. 1998; Maynard et al. 1999; Reich et al. 2001b; Zohary et al. 1994) can increase or decrease the mutual information between population responses and stimuli compared with that expected when the neurons are independent (Abbott and Dayan 1999; Anderson et al. 2007; Averbeck and Lee 2004; Franco et al. 2004; Michel and Jacobs 2006; Nirenberg and Latham 2003; Oram et al. 1998; Panzeri et al. 1999; Schneidman et al. 2006; Wilke and Eurich 2001, 2002).

In this article, I describe the dynamics of the statistics of neuronal responses induced by visual stimuli in the anterior superior temporal sulcus (STSa) of the macaque monkey and quantify their impact on the information carried by the responses. Stimulus-elicited activity starts with a brief period in which the trial-by-trial variability of neuronal activity is reduced and independent of previous activity. The between-cell correlation of the trial-by-trial variability also shows a decrease at response onset, coincident with the rise in the signal correlation. Extension of previous modeling work (Oram et al. 1999, 2001) suggests that these phenomena are related and can be explained by a dual-process model in which the activitygenerating process undergoes a "reset" at response onset. The results are discussed in the context of neuronal output activity being governed by recurrent activity in the "prestimulus" period, then changing at the onset of the stimulus-elicited response to being largely dominated by feedforward connections. Thus the study of the dynamics of response statistics informs us about the neural code, helps discriminate models of response statistics, and helps elucidate the underlying circuits (Oram et al. 2007; Smith and Kohn 2008).

\section{METHODS}

Responses elicited by a wide range of visual stimuli, including faces, bodies, hands, animals, and abstract images from single and pairs of single neurons in the superior temporal sulcus of two macaque monkeys were collected. The experimental protocols have been previously described (Foldiak et al. 2003; Oram et al. 2002; van Rossum et al. 2008). Extracellular single-unit recordings were made using standard techniques for single electrode recording from the upper and lower banks of the anterior part of the superior temporal sulcus (STSa) of two monkeys (Macaca mulatta) performing a visual fixation task. Briefly, recording chambers were implanted under pentobarbitol (Sagatal) anesthesia with full sterile precautions over both hemispheres to enable electrode penetrations to reach the STSa. Cells were recorded using a single tungsten-in-glass microelectrode inserted through the dura mater. The electrical signal was amplified (Neurolog NL104) and then filtered using a $50-\mathrm{Hz}$ notch filter together with low-pass (20 $\mathrm{kHz}$ ) and high-pass $(300 \mathrm{~Hz})$ filters (Neurolog NL125). The subject's eye position $\left( \pm 1^{\circ}\right.$ ) was monitored (IView, SMI, Kolding, Germany). A Pentium IV PC with a CED 1401 interface running Spike2 (Cam- bridge Electronic Design [CED], Cambridge, UK) recorded eye position, spike arrival, and stimulus on/offset times. The animal procedures were conducted under UK Home Office project license (PPL 60/3186) and approved by the Animal Welfare and Ethical Committee of the University of St Andrews.

The subject received a drop of fruit juice reward every $500 \mathrm{~ms}$ of fixation $\left( \pm 3^{\circ}\right)$ while static stimuli $\left(19 \times 19^{\circ}\right)$ were displayed. During initial screening, 24-bit color images of different perspective views of monkey and human head, animals, fractal patterns, natural scenes, and everyday objects were presented (22-41 stimuli; Edwards et al. 2003). Visual stimuli were presented centrally on a black monitor screen (Sony GDM-20D11, resolution 25.7 pixels/degree, refresh rate $72 \mathrm{~Hz}$ ), $57 \mathrm{~cm}$ from the subject in a random sequence for $333 \mathrm{~ms}$ with a 333-ms interstimulus interval.

Spikes were recorded during the period of fixation. If the subject looked away for $>100 \mathrm{~ms}$, both spike recording and presentation of stimuli stopped until the subject resumed fixation for $\geq 500 \mathrm{~ms}$. The results from initial screening were used to select stimuli that elicited large responses from the neuron (effective stimuli) and to select stimuli that elicited small or no response (ineffective stimuli). For different neurons effective and ineffective stimuli included different views of the head (Perrett et al. 1991), abstract patterns, and familiar objects (Foldiak et al. 2003). Details of the stimulus selectivity of these neurons has been reported elsewhere (Barraclough et al. 2005, 2006, 2009; Edwards et al. 2003; Foldiak et al. 2003; Oram et al. 2002; van Rossum et al. 2008). The anterior-posterior extent of the recorded cells was from 7 to $10 \mathrm{~mm}$ anterior of the interaural plane, consistent with previous studies showing visual responses to static images in the STSa (Baylis and Rolls 1987; Bruce et al. 1981; Perrett et al. 1982, 1991).

\section{Data analysis}

Off-line isolation of single neurons was performed using a template matching procedure and principal components analysis on the continuous recording (Spike2; CED). Each neuron's response to a stimulus was calculated by aligning spike times on each occurrence of that particular stimulus (trials). For each neuron a poststimulus time histogram was generated and a spike density function (SDF) calculated for each stimulus by summing across trials (bin size $=1 \mathrm{~ms}$ ) and smoothing (Gaussian kernel, $\sigma=10 \mathrm{~ms}$ ). Background or spontaneous activity was measured in the $200-\mathrm{ms}$ period prior to stimulus onset. Data from a neuron were accepted if at least five trials were obtained per stimulus (median $=19$, interquartile range $=11.29)$ and the peak response (measured in the $100 \mathrm{~ms}$ after response latency) to at least one stimulus was $>5$ spikes/s above background activity.

\section{Single-cell analysis}

Analysis of the data from a single cell is shown schematically in Fig. 1. The variance of the responses was related to the mean (e.g., in F1 the period 70-270 ms poststimulus onset, $\ln \left(\sigma^{2}\right)=0.17+1.05 \times$ $\left.\ln (\mu), r^{2}=0.79, P<0.00001\right)$. The variability of the responses was therefore assessed using the Fano factor (variance/mean of spike count), $\mathrm{FF}_{\text {stim }}$

$$
\mathrm{FF}_{\text {stim }}=\frac{\sigma_{\text {stim }}^{2}}{\mu_{\text {stim }}}
$$

where $\mu_{\text {stim }}$ and $\sigma_{\text {stim }}$ are the mean and SD of the spike counts to stimulus (stim). The $\mathrm{FF}_{\text {stim }}$ values were aligned to the center point of each bin (Fig. 1A, dashed lines). The Fano factor averaged across stimuli gives the neuron Fano factor $\left(\mathrm{FF}_{\text {neuron }}\right)$, the average of the $\mathrm{FF}_{\text {neuron }}$ giving the population estimate $(\mathrm{FF})$.

The sequential or autocorrelation was assessed for each cell/stimulus combination using Pearson's correlation coefficient between two successive, nonoverlapping sample windows. The sequential correla- 

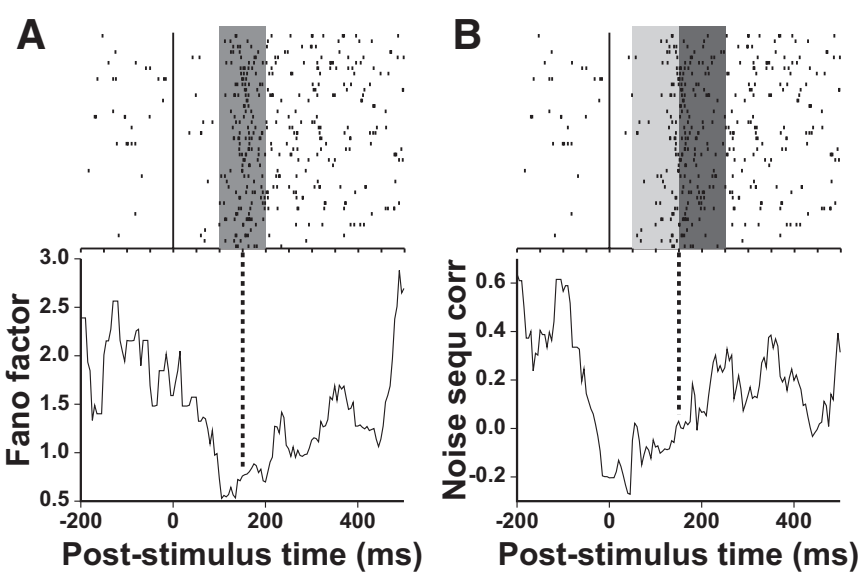

FIG. 1. Measurement of single-cell response statistics. Top panels: rastergrams of the responses of a single cell to a single stimulus. A: the Fano factor from a window of $100 \mathrm{~ms}$ (top, shaded area) was calculated every $5 \mathrm{~ms}$. The estimate was assigned to the midpoint of the window (dashed line). $B$ : the sequential correlation of the trial-by-trial variability (noise) was assessed using spike counts in 2 nonoverlapping windows (top, light and dark gray areas). The resultant estimate was assigned to the time of the start of the second window (dashed line).

tion is broken down into the correlation of the signal $\left(r_{\text {auto signal }}\right)$ and correlation of the noise ( $r_{\text {auto_noise }}$, Fig. $1 B$ ), corresponding to the correlation between successive sample windows of the mean response to each stimulus and the correlation between the successive sample windows of the trial-by-trial variability about those means.

The signal correlation of each neuron $\left(r_{\text {neuron_auto_signal }}\right)$ is the correlation between the mean spike counts of successive sample windows across stimuli

$$
r_{\text {neuron_auto_signal }}=\frac{\sum_{\mathrm{stim}}\left(\mu_{\mathrm{w} 1}-\overline{\mu_{\mathrm{w} 1}}\right)\left(\mu_{\mathrm{w} 2}-\overline{\mu_{\mathrm{w} 2}}\right)}{\sqrt{\sum_{\mathrm{stim}}\left(\mu_{\mathrm{w} 1}-\overline{\mu_{\mathrm{w} 1}}\right)^{2} \sum_{\mathrm{stim}}\left(\mu_{\mathrm{w} 2}-\overline{\mu_{\mathrm{w} 2}}\right)^{2}}}
$$

where $\mu_{\mathrm{w} 1}$ and $\mu_{\mathrm{w} 2}$ are the mean spike counts in window 1 and window 2 to each stimulus stim and $\overline{\mu_{\mathrm{w} 1}}$ and $\overline{\mu_{\mathrm{w} 2}}$ are the mean spike counts across stimuli of window 1 and window 2 , respectively.

The simple average of $r_{\text {neuron_auto_signal }}$ will result in an underestimate of the true value of the population estimate $r_{\text {auto signal }}$ because of the skewed distribution of correlation coefficients (Snedecor and Cochran 1980). Instead, the average of Fisher's transformation of $r_{\text {neuron_auto_signal }}$

$$
z_{\text {neuron_auto_signal }}=\frac{1}{2} \ln \left(\frac{1+r_{\text {neuron_auto_signal }}}{1-r_{\text {neuron_auto_signal }}}\right)
$$

gives a population estimate $z_{\text {auto_signal }}$, which can be inverse transformed to obtain a better estimate of the population sequential correlation of the signal $r_{\text {auto_signal }}$; however, the Fisher transformation can result in a small overestimate of the true value of the correlation (Snedecor and Cochran 1980): the results reported here are also seen using the (under)estimate from the average of $r_{\text {neuron_auto_signal. }}$.

The correlation of the trial-by-trial variability between two nonoverlapping windows can be calculated to each stimulus (Fig. 1B). These values can then be averaged (using Fisher's transform) to obtain an estimate of the sequential correlation of the noise for the neuron. However, such a procedure does not work well with small windows if either window has no measurable variability (i.e., no spikes) in the limited number of trials. To allow calculation of the sequential correlation of the trial-by-trial variability of each neuron $\left(r_{\text {neuron_auto_noise }}\right)$ for small windows, the spike counts were collapsed across all stimuli by transforming each response to each stimulus $\mathrm{s}\left(r_{s}\right)$ into its $z$-score, $r_{z}=\left(r_{s}-\bar{r}_{s}\right) / \sigma_{s}$, where $\bar{r}_{s}$ is the mean and $\sigma_{s}$ is the SD of the responses to stimulus $s$, setting $r_{z}=0$ when $\sigma_{s}=0$. Without this normalization, the measure of noise correlation assessed over all trials will be artifactually high because of the inclusion of the impact of the stimulus (signal). The correlation of the $r_{z}$ between windows w 1 and $\mathrm{w} 2$ for each neuron

$$
r_{\text {neuron_sequ_noise }}=\frac{\sum_{\text {trials }}\left(r_{z \mathrm{w} 1}-\overline{r_{z \mathrm{~W} 1}}\right)\left(r_{z \mathrm{w} 2}-\overline{r_{z \mathrm{w} 2}}\right)}{\sqrt{\sum_{\text {trials }}\left(r_{z \mathrm{w} 1}-\overline{r_{z \mathrm{w} 1}}\right)^{2} \sum_{\text {trials }}\left(r_{z \mathrm{~W} 2}-\overline{r_{z \mathrm{~W} 2}}\right)^{2}}}
$$

is thus the sequential correlation of the normalized trial-by-trial response variability about the mean and does not reflect correlation of the mean response (signal), nor is it biased toward those stimuli with greater absolute response variability (Lee et al. 1998; Oram et al. 2001). Averaging $r_{\text {neuron_auto_noise }}$ across neurons, using Fisher's transformation

$$
z_{\text {neuron_sequ_noise }}=\frac{1}{2} \ln \left(\frac{1+r_{\text {neuron_sequ_noise }}}{1-r_{\text {neuron_sequ_noise }}}\right)
$$

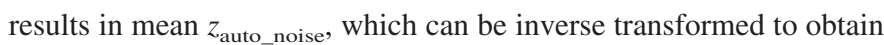
an estimate of the population sequential correlation $r_{\text {auto_noise }}$.

\section{Between-cell analysis}

The recordings allowed separation of pairs, three and in two cases four isolated neurons from the same electrode (Spike2, CED; principal component analysis based algorithm; minimum Mahalanobis distance $=1.5$ ). Recordings in which the waveforms drifted over time were rejected. All separated spike clusters were then examined visually and only if the separation was clear (i.e., no "unclassified" spike waveforms lay between the clusters) were the isolated neurons accepted.

The correlation between the responses of pairs of neurons can also be described using the signal correlation, $r_{\text {cross signal }}$, and the "noise" correlation, $r_{\text {cross_noise }}$ (Gawne and Richmond 1993). The signal correlation for each neuron ( $\left.r_{\text {neuron_cross_signal }}\right)$ is the correlation between the mean spike counts to each stimulus

$$
r_{\text {neuron_cross_signal }}=\frac{\sum_{\text {stim }}\left(\mu_{1}-\overline{\mu_{1}}\right)\left(\mu_{2}-\overline{\mu_{2}}\right)}{\sqrt{\sum_{\text {stim }}\left(\mu_{1}-\overline{\mu_{1}}\right)^{2} \sum_{\text {stim }}\left(\mu_{2}-\overline{\mu_{2}}\right)^{2}}}
$$

where $\mu_{1}$ and $\mu_{2}$ are the mean spike counts of neuron 1 and neuron 2 to each stimulus; $\overline{\mu_{1}}$ and $\overline{\mu_{2}}$ are the mean across stimuli of neuron 1 and neuron 2, respectively. The Fisher's transformed $r_{\text {neuron_cross_signal }}$ were averaged and inverse transformed to obtain a population-based estimate, $r_{\text {cross_signal }}$. The trial-by-trial or noise correlation between neurons was calculated in an analogous manner to the calculation of the $r_{\text {auto_noise }}$. The cross-correlation $r_{\text {cross_noise }}$ used the $z$-scored firing rates, calculating the correlation of the trial $z$-scores from all stimuli. Combining of data across stimuli is supported by the observation that the trial-by-trial or noise cross-correlation does not vary with stimulus (Kohn and Smith 2005).

The values of FF, $r_{\text {auto_signal }}, r_{\text {auto_noise }}, r_{\text {cross_signal }}$, and $r_{\text {cross_noise }}$ were calculated every $5 \mathrm{~ms}$ using sample windows of duration 5,10 , $25,50,100$, and $200 \mathrm{~ms}$. Estimates obtained of FF, $r_{\text {auto_signal, }}$, $r_{\text {auto_noise }}, r_{\text {cross_signal }}$, and $r_{\text {cross_noise }}$ every $5 \mathrm{~ms}$ are not independent when the window size is $>5 \mathrm{~ms}$. Statistical analyses used nonoverlapping, independent samples from each neuron, but data are displayed every $5 \mathrm{~ms}$ to aid visual interpretation of these summary statistics.

\section{Modeling}

The three models used are shown schematically in Fig. 2. All three F2 models use the spike density function (SDF, Fig. 2, top) as the starting point. The nonhomogeneous Poisson process (NHPP) model used independent binomial processes with 1-ms time bins, the SDF value giving the probability $p$ of a spike occurring in that time bin (Fig. 2, middle row, left). The effects of non-Poissonian spike count distribution, particularly with respect to correlations, are critically dependent 

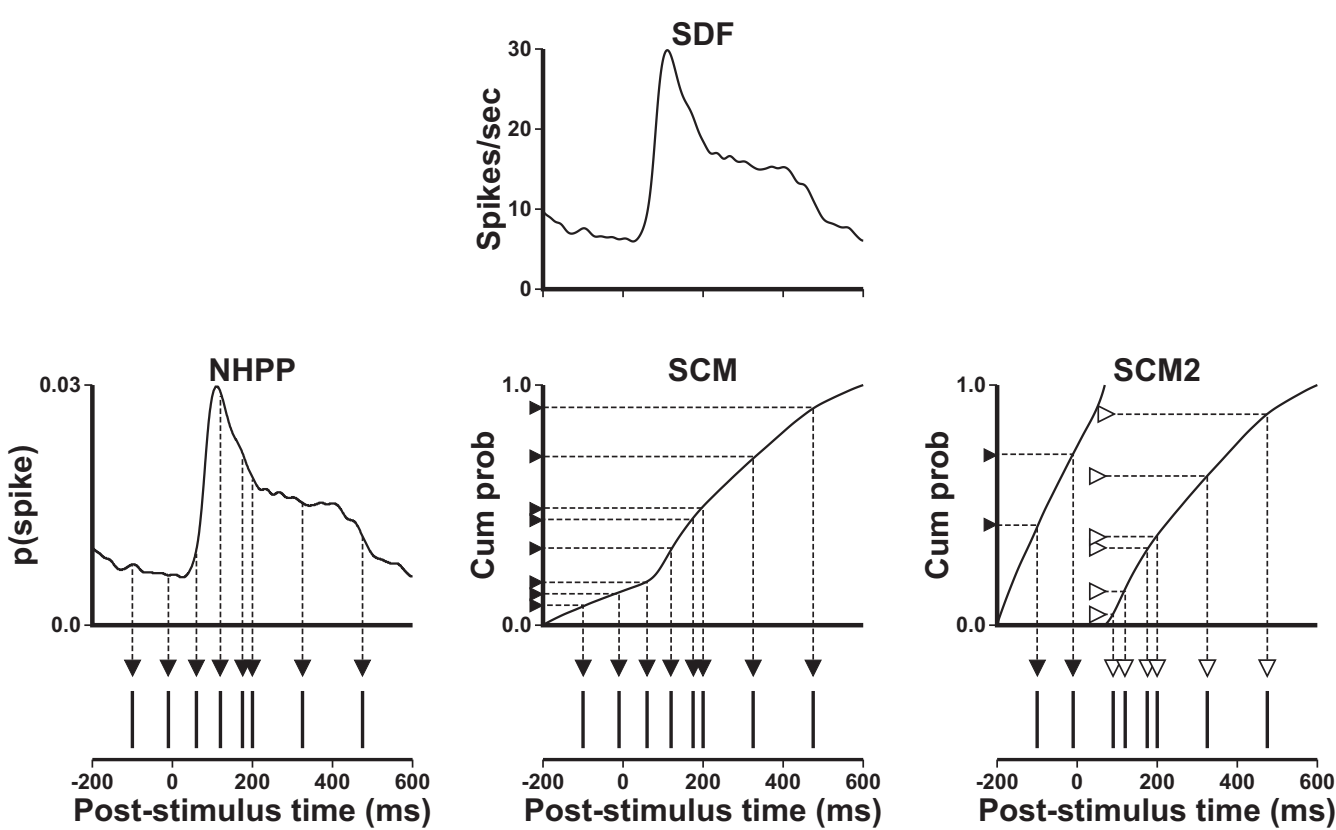

FIG. 2. Modeling spike trains. The 3 models all use spike density function (SDF, top) of a neuron's responses. To obtain modeled spike trains using the nonhomogeneous Poisson process (NHPP), the SDF can be normalized to give the probability of a spike $p$ (spike), in each millisecond (middle row, left). If a random number [0...1] is $<p$ (spike), then a spike is allocated into the spike train (dashed lines, solid arrow to bottom left). In the spike count matched model (SCM, middle; Oram et al. 1999, 2001), the SDF is converted to the cumulative spike density function (CSDF). For each spike observed in the spike train (here 8 spikes), a uniform random number [0...1] is transformed into a spike time by the inverse of the CSDF (solid arrows, dashed lines, bottom middle). In the dual-process SCM model (SCM2, right), the response period is split into 2 sections at $70 \mathrm{~ms}$, the typical latency of inferotemporal cortex and superior temporal sulcus (IT/STS) neurons. The SCM model is then run for the initial period [ $-200 \ldots 69 \mathrm{~ms}]$ (dashed line, solid arrows) and, separately, for the period [70. . .600 $\mathrm{ms}$ ( dashed line, open arrows).

on the precise spike counts (Oram et al. 1999). The spike count matched (SCM) model (Oram 2005; Oram et al. 1999, 2001, 2002) was used to match the observed coarse temporal statistics as closely as possible. Briefly, for each cell, the SDF is transformed into a cumulative spike density function (CSDF, Fig. 2, middle) for each stimulus at each time point $t\left[\sum_{i=1}^{t} \mathrm{SDF}(i)\right]$. Normalization by the value at the end of the sample period $(t=T)$ gives the cumulative spike probability function $\operatorname{CSPF}(t)=\operatorname{CSDF}(t) / \operatorname{CSDF}(T)$. The spike times of each artificial trial are found by applying the inverse of the cumulative probability distribution to random numbers in the interval $(0,1)$, $R[0 . .1]$ (Fig. 2, middle, black arrows). The time bin (width $\delta t$, here $1 \mathrm{~ms})$ in which a spike occurs, $t_{\text {spike, }}$, is such that $t_{\text {spike }}$ satisfies $\operatorname{CSPF}(k) \leq R[0 \ldots 1]<\operatorname{CSPF}(k+1)$, the time of the $k$ th bin being $k \delta t-(k+1) \delta t$.

When generating surrogate data for pairs of neurons using the SCM model (Oram et al. 2001), each pair of spike count distributions and their correlation are preserved by stepping through the experimental data trial by trial and forcing each simulated trial of each cell to have the same number of spikes as the corresponding experimental trial. The SCM model therefore incorporates the slow variation in firing rate and the distribution of spike counts generated by individual neurons as well as the coarse temporal correlation of the spike counts and the correlation between the spike density functions (i.e., correlation between the slow variation in firing rate of the individual neurons over time; for details see Oram et al. 2001).

The SCM model simulates spike trains assuming a single process (here running from $200 \mathrm{~ms}$ before to $600 \mathrm{~ms}$ poststimulus onset). SCM2, a dual-process extension, is modeled by applying a "reset." This is achieved by running the SCM, including the normalizations to generate a CSDF for the period -200 to $69 \mathrm{~ms}$. The SCM model is then rerun, with recalculation of the CSDF, using the data from 70 to $600 \mathrm{~ms}$ poststimulus onset (Fig. 2, middle row, right). Therefore in the SCM2 model the spike counts are matched on a trial-by-trial basis separately for the two different intervals (Fig. 2, middle row, right: solid arrows for period -200 . .69 ms, open arrows for period
$70 . .600 \mathrm{~ms}$ ). The reset or change of spike-generation process at 70 ms was chosen to approximate the response latency of STS/IT neural populations (Eifuku et al. 2004; Kiani et al. 2005; Oram and Perrett 1992, 1996; Sary et al. 2006). Thus the SCM2 model can be interpreted as assuming a "nonstimulus driven" and a separate (independent) "stimulus driven" spike-generation process. An absolute refractory period of $1 \mathrm{~ms}$ was applied to the SCM and SCM2 models. To control for potential biases due to limited sampling, the number of trials from each neuron was also matched. The spike trains from the NHPP, SCM, and SCM2 models were analyzed using the same methods as used for the experimental data.

\section{RESULTS}

Visually elicited responses from 74 neurons from two rhesus macaque monkeys were analyzed following presentation of stimuli for $333 \mathrm{~ms}$. In all, 51 neurons were tested with effective $(>70 \%$ of maximum) and ineffective $(<30 \%$ of maximum response) stimuli. The 74 neurons allowed for 67 pairs of simultaneously recorded neurons to be examined.

\section{Response statistics of single neurons}

An example of the calculation of the Fano factor for a single neuron/stimulus combination $\left(\mathrm{FF}_{\text {stim }}\right)$ using 100-ms windows is shown in Fig. 1A. Across the population, the average Fano factor (see METHODS) was significantly $>1$ at all time points for sample windows of $\geq 10 \mathrm{~ms}$ (Fig. 3, top row). As expected, the F3 Fano factor increases with window size (Baddeley et al. 1997; Buracas et al. 1998; Carandini 2004; de Ruyter van Steveninck et al. 1997; Osborne et al. 2004; Teich et al. 1996).

Figure $3 B$ allows comparison of the time course of the Fano factor averaged across the 74 neurons using 100-ms windows 

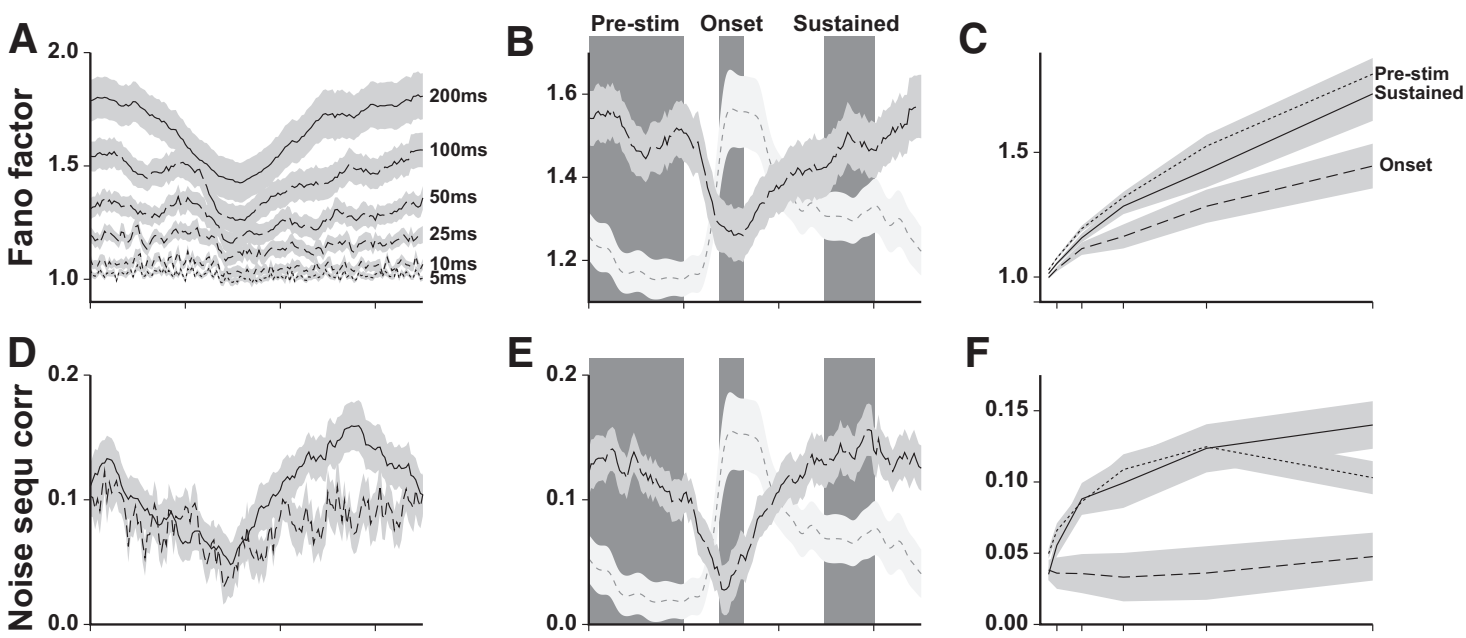

E

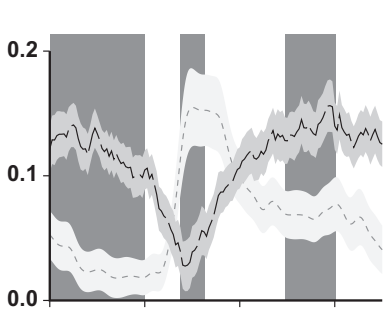

$\mathbf{F}$

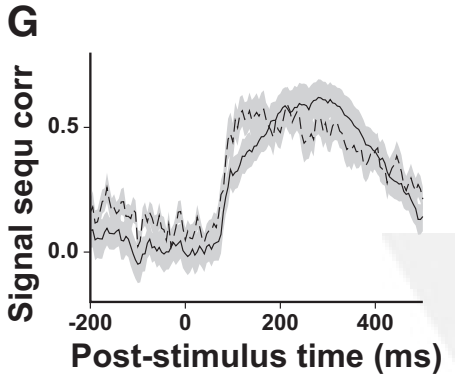

H

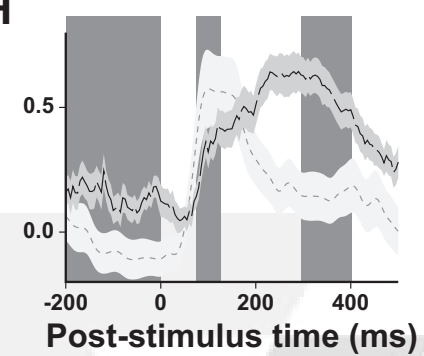

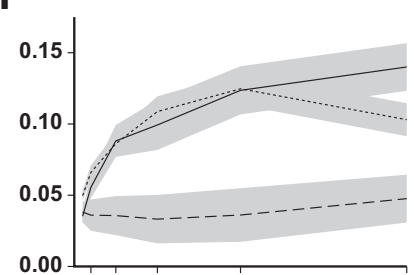

I

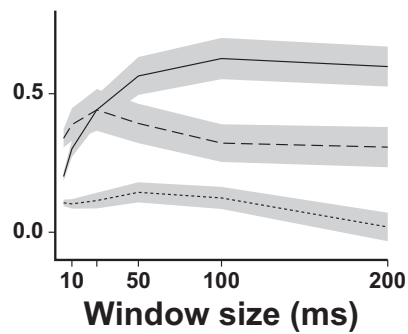

FIG. 3. Response statistics of single neurons assessed using varying window sizes. Sequential response statistics averaged across 74 single neurons (see

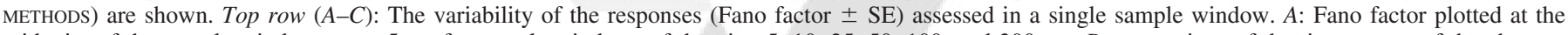

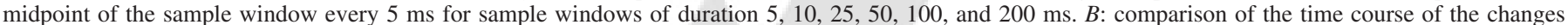

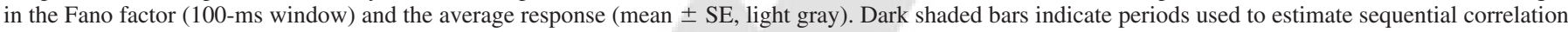

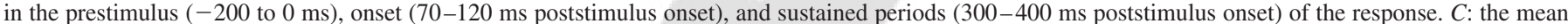

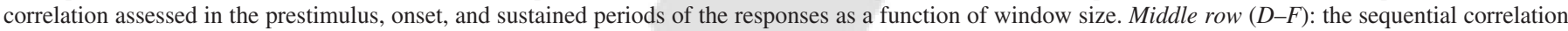

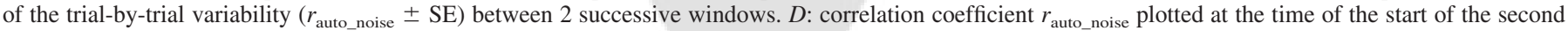

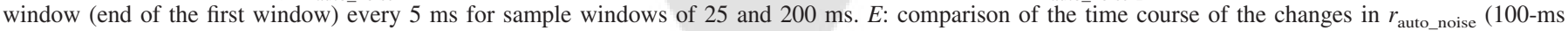

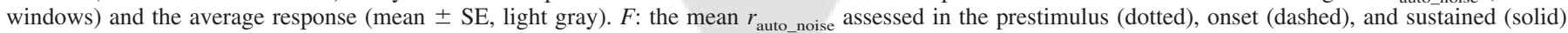

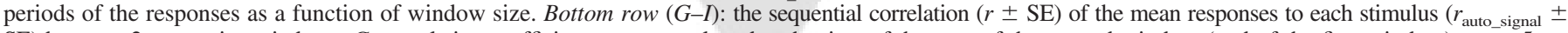

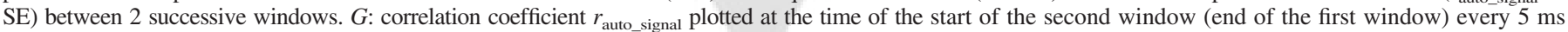

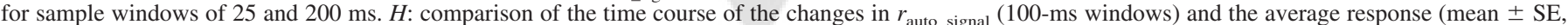
light gray). $I$ : the mean $r_{\text {auto_signal }}$ assessed in the prestimulus, onset, and sustained periods of the responses as a function of window size.

with the time course of the neuronal activity (average spike density function, gray). There is a transient dip in Fano factor at the onset of neuronal responses (Amarasingham et al. 2006; Churchland et al. 2006; Osborne et al. 2004; Uka and DeAngelis 2003). The time of the minima estimated from different window sizes ranged from 80 to $140 \mathrm{~ms}$ poststimulus onset, approximately the time of peak response. The dip in the Fano factor at about $70 \mathrm{~ms}$ is significant (ANOVA, $P<0.005$ ) for window sizes down to $10 \mathrm{~ms}$ and a trend $(P=0.08)$ is present for 5-ms windows. Detection of the dip in normalized variability down to such short sample windows suggests a rapid underlying mechanism.

An example of the sequential autocorrelation of the trial-bytrial variability assessed between adjacent bins, $r_{\text {auto noise stim }}$, is shown in Fig. $1 B$ for the same cell/stimulus as in Fig. $1 A$. Note the marked decrease (dip) in the correlation around response onset, in this case showing negative correlation. The average sequential correlation of the trial-by-trial variability ( $\left.r_{\text {auto noise }}\right)$ is shown in Fig. 3, $D-F$. Like the Fano factor, $r_{\text {auto_noise }}$ shows a dip at about $70 \mathrm{~ms}$ and increases systematically with window size, reaching a stable value at window sizes of nearly $100 \mathrm{~ms}$ (Fig. 3, D and F). The average sequential correlation for windows $\geq 100 \mathrm{~ms}$ in both the prestimulus period and the sustained period $(0.13 \pm 0.02)$ are similar to values reported previously $(0.14-0.16)$ in parietal cortex, MT, IT, and motor cortices (Bair et al. 2001; Lee et al. 1998; Oram et al. 2007; Uka and DeAngelis 2003), although not as high as found in some studies (e.g., 0.4 in MT; Osborne et al. 2004). These previous findings are extended here by showing that the dip in $r_{\text {auto noise }}$ around response onset (Fig. $3 F)$ is significant for window lengths of $\geq 25 \mathrm{~ms}(P<0.005$, each comparison), with a trend $(P=0.09)$ for windows of length $10 \mathrm{~ms}$. As with the dip in the Fano factor, detection of the dip in $r_{\text {auto_noise }}$ in short sample windows suggests a rapid underlying mechanism.

The sequential (auto-) correlation of the "signal," assessed using the mean response to each stimulus in two adjacent bins, $r_{\text {auto_signal }}$ is shown in Fig. 3, $\left.G-I\right)$. Combined with the presence of sequential correlation of the noise $\left(r_{\text {auto_noise }}>0\right)$, the small numerical differences in the mean prestimulus response estimates due to limited sampling are maintained in time, giving rise to nonzero estimates of $r_{\text {auto_signal }}$. Although $r_{\text {auto_signal }}$ does not vary with the size of the sample window in the prestimulus $(P=0.22)$ and onset periods $(P=0.54$, see 

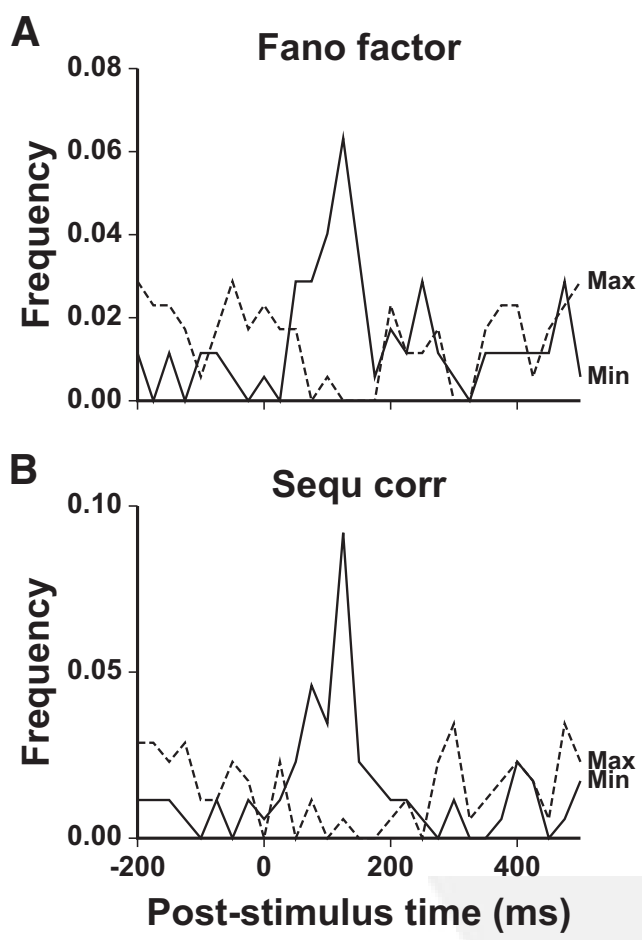

FIG. 4. Timing of minimum and maximum Fano factor and sequentialcorrelation. The time of the maximum (dashed lines) and minimum (solid lines) Fano factor (top) and sequential correlation of the trial-by-trial variability, $r_{\text {auto_noise }}($ bottom) of each neuron is plotted as a frequency distribution. Minimum values cluster in the period $50-150 \mathrm{~ms}$ for both measures. Maxima occur in the time periods outside this.

Fig. 3I), $r_{\text {auto signal }}$ does vary with sample window during the sustained period (ANOVA, $P<0.001$ ).

Reductions in the average sequential correlation $r_{\text {auto noise }}$ and Fano factor around response onset are evident cell by cell. Figure 4 plots the frequency distribution of the time for the minimum (solid line) and maximum (dashed line) of the $r_{\text {auto noise (Fig. 4, top) and Fano factor (Fig. 4, bottom) of each }}$ neuron assessed using 100-ms windows. The clustering of the minima of both $r_{\text {auto noise }}$ and the Fano factor in the period $75-175$ was significant for all window sizes $\left(\chi^{2}, P<0.05\right.$, each comparison). If the clustering were due to increased variability of the estimates of $r_{\text {auto_noise }}$ and the Fano factor, it would be expected that the maxima would also cluster in this period. However, the maxima tend to occur outside this period ( $r_{\text {auto_noise }}$ : window sizes 50 and $100 \mathrm{~ms}, P<0.01$; window sizes 10 and $25 \mathrm{~ms}, P<0.1$; Fano factor: window sizes 5 and $25-200 \mathrm{~ms}, P<0.05)$.

It is also of interest to know whether the response statistics vary with response amplitude or stimulus effectiveness. The $r_{\text {autonnoise }}$ and Fano factor observed in responses to stimuli that elicited large $(>70 \%$ of maximum) and small $(<30 \%$ of maximum) responses were examined separately (Fig. 5). [Splitting the data into groups by stimuli that elicit either large or small mean responses removes any meaningful assessment of $r_{\text {auto_signal. }}$. The average spike density function (Fig. 5, inset) shows that there are negligible responses to ineffective stimuli and strong responses to effective stimuli. The consistency of $r_{\text {auto_noise }}$ in the prestimulus period (effective: $0.15 \pm 0.03$; ineffective: $0.13 \pm 0.02$ ), during onset (effective: $0.02 \pm 0.04$; ineffective: $0.05 \pm 0.03$ ) and sustained period (effective:
$0.18 \pm 0.04$; ineffective: $0.12 \pm 0.03$ ) indicates that the dip in sequential correlation is not attributable to changes in response amplitude. The dip in mean $r_{\text {auto noise }}$ was present in the responses elicited by ineffective (and effective) stimuli for windows as small as $10 \mathrm{~ms}$, although statistically significant $(P<0.05)$ only for windows of $\geq 25 \mathrm{~ms}$. The Fano factor in the prestimulus period (effective: $1.5 \pm 0.1$; ineffective: $1.5 \pm$ 0.1 ), during onset (effective: $1.3 \pm 0.2$; ineffective: $1.3 \pm 0.1$ ) and sustained period (effective: $1.5 \pm 0.1$; ineffective: $1.4 \pm$ 0.1 ) indicates that the dip in Fano factor at response onset (Fig. 5 , top) is not attributable to changes in response amplitude. The transient "dip" in Fano factor was statistically significant $(P<$ 0.05 ) for window sizes down to $10 \mathrm{~ms}$ in the responses elicited by both effective and ineffective stimuli.

Although the presence of a dip in sequential correlation and Fano factor around response onset cannot be attributable to differences in response magnitude, the time course of the response statistics varies with response magnitude, being somewhat faster to "recover" to prestimulus levels for effective than ineffective stimuli (Fig. 5). For example, although the times of the minima of the sequential correlation are almost identical $(90 \mathrm{~ms}$ for ineffective stimuli; $95 \mathrm{~ms}$ for effective stimuli), the recovery to prestimulus levels takes only $60 \mathrm{~ms}$ with effective stimuli compared with $185 \mathrm{~ms}$ with ineffective

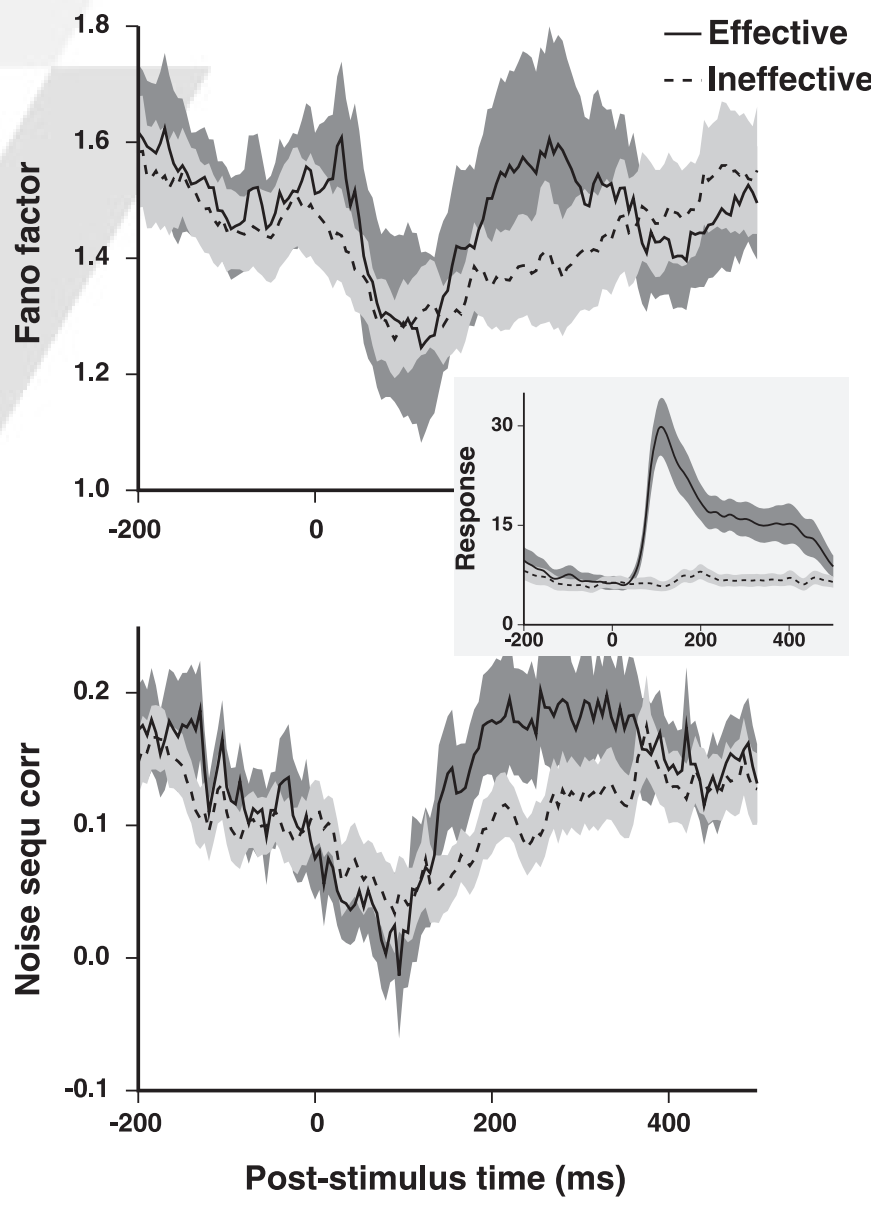

FIG. 5. Transient decrease in Fano factor and sequential-correlation to effective and ineffective stimuli. The average Fano factor (top) and $r_{\text {auto_noise }}$ (bottom) assessed using 100-ms window for 51 neurons where responses to both effective (solid line, dark gray $=\mathrm{SE}$ ) and ineffective (dashed line, light gray $=\mathrm{SE}$ ) stimuli were obtained. Inset shows the average spike density functions to the effective and ineffective stimuli. 

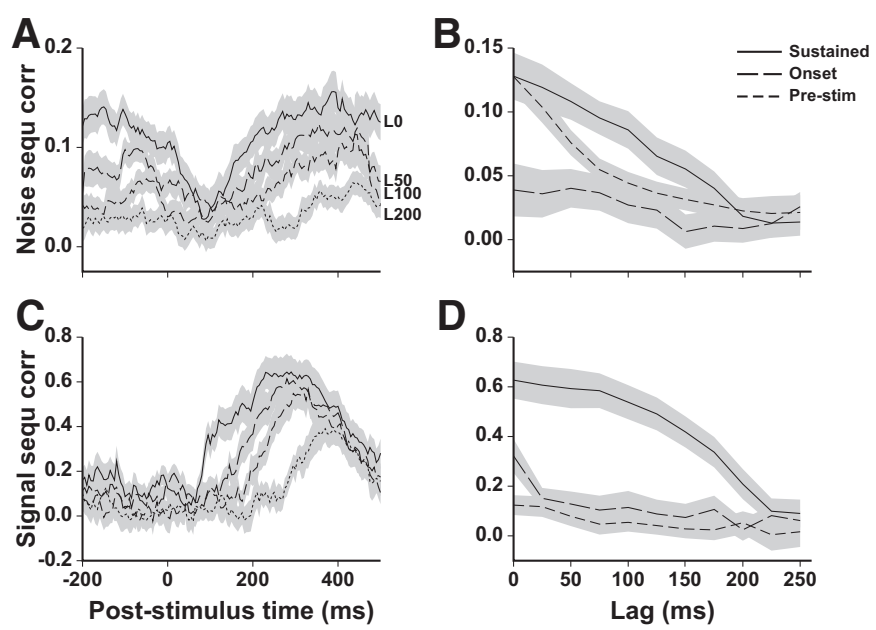

FIG. 6. Decay of sequential correlation. $A$ : the mean sequential correlation of the trial-by-trial variability ( $r_{\text {auto noise }} \pm$ SE) assessed using 100-ms windows is plotted as a function of poststimulus onset time for delays or lags between the sample windows of 0, 50, 100, 150, and $200 \mathrm{~ms}$ (solid, long dashed, dashed, short-dashed, and dotted lines, respectively). Values are plotted using the start of the later window. Thus the correlation of the data from window (100..200) at lag $200 \mathrm{~ms}$ corresponds to the window $(-150 \ldots-100)$, lag $100 \mathrm{~ms}$ corresponds to window $(-100 \ldots 0)$, lag $50 \mathrm{~ms}$ $(-50 \ldots 50)$, and lag $0(0 \ldots 100)$. All these lags are all aligned at time 100 . $B: r_{\text {auto-noise }}( \pm \mathrm{SE})$ as a function of the lag between $100-\mathrm{ms}$ sample windows for the prestimulus $(-200$. .0 ms, dotted line), onset ( $70 \ldots 120 \mathrm{~ms}$ poststimulus, dashed line), and sustained (300 . .400 ms poststimulus, solid line). $C$ : the sequential correlation of the mean responses ( $\left.r_{\text {auto signal }} \pm \mathrm{SE}\right)$ assessed using 100-ms windows for lags of 0 to $200 \mathrm{~ms}$ between the sample windows (line coding as in $A$ ). $D$ : $r_{\text {auto signal }}( \pm \mathrm{SE}$ ) as a function of the lag between 100 -ms sample windows (line coding as in $B$ ).

stimuli. The slower recovery to prestimulus levels in the responses to ineffective stimuli is also seen with the Fano factor. Thus both response variability and sequential correlation of neuronal activity are reduced at around the time when neurons in STSa become active, even in neurons that are not strongly activated by the input stimulus. Although the magnitude and time of the "dip" are similar to both effective and ineffective stimuli, the effect is shorter lasting in responses to effective than that in response to ineffective stimuli.

Next, I extend previous studies by examining the impact of lag between sample windows. With a constant spike-generating process the correlation between successive short sample windows will be independent of the lag between two sample windows. Now consider a spike-generating process that operates at short timescales. Between time point 1 and time point 2 , we can explain $R^{2}$ of the variance at time point 2 based on the data at time point 1 . Similarly, between time points 2 and 3 we can explain $R^{2}$. Assuming no higher-order correlation, we can therefore explain $R^{2} \times R^{2}$ of the variance of the response at time point 3 based on the values at time point 1 . Given that $R^{2}$ is necessarily $\leq 1$, this gives an exponential decay in the $R^{2}$ (and the correlation coefficient $r$ ) as the lag is increased. Thus if the spike-generating process operates at (multiple) short timescales, the correlation between successive sample windows will decrease exponentially as the lag between the two sample windows is increased.

With a 100-ms sample window (Fig. 6), the magnitude of the F6 sequential correlation of the trial-by-trial variability $\left(r_{\text {auto }}\right.$ noise $)$ decreases with increasing lag (Fig. 6, $A$ and $B$ ). The "dip" in $r_{\text {auto_noise }}$ is numerically present for lags $\leq 225 \mathrm{~ms}$ (Fig. 5A), being statistically significant $(P<0.05)$ for lags $\leq 75 \mathrm{~ms}$ and showing a trend $(P<0.1)$ for lags $\leq 150 \mathrm{~ms}$. The exponential decay of $r_{\text {auto_noise }}$ with lag is evident for all sample window sizes (see Table 1).

The time constant for the decay of $r_{\text {auto noise }}$ with increasing lag between the sample windows obtained from the prestimulus data $(107 \pm 6 \mathrm{~ms})$ was less than that in both the onset $(188 \pm 50 \mathrm{~ms})$ and the sustained periods of the response (143 \pm 17

TABLE 1. Decrease in sequential correlation, sequential correlation of the noise, and sequential correlation of the signal

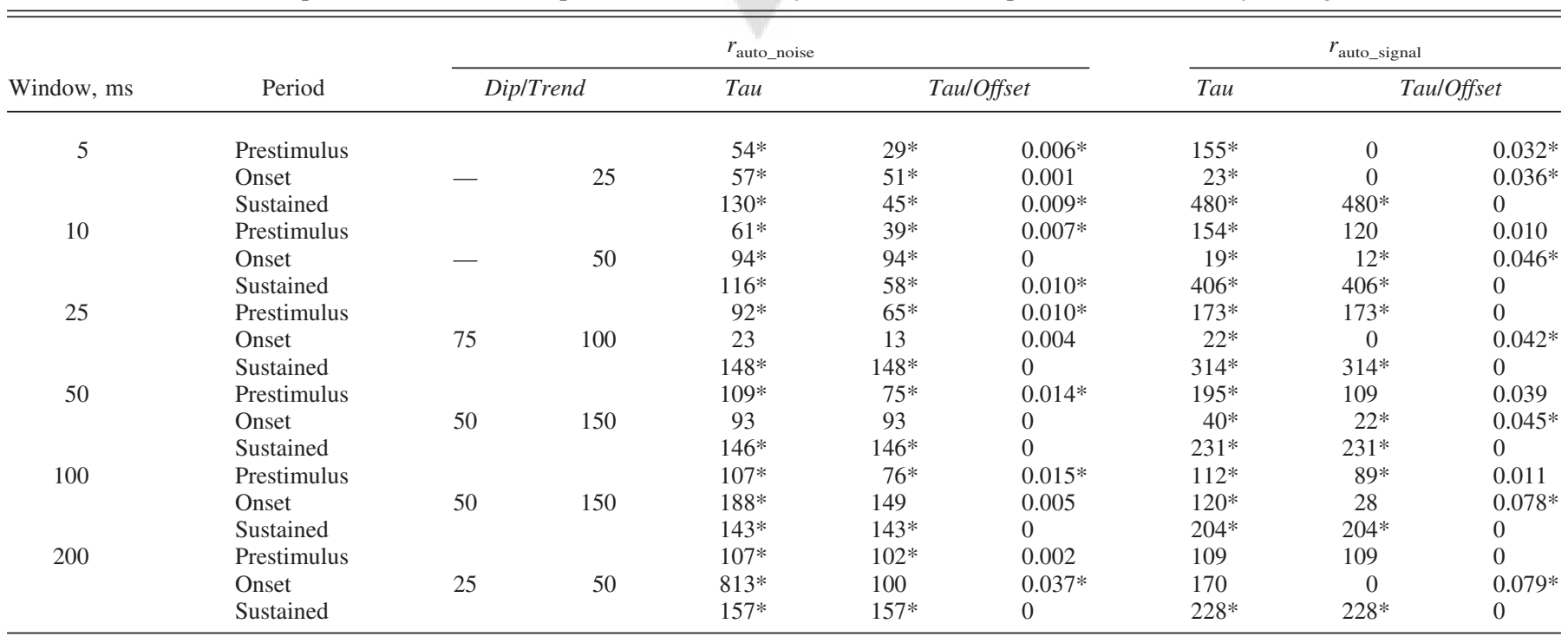

With respect to decrease in sequential correlation: Measures of the decrease in the sequential correlation of the trial-by-trial variability $\left(r_{\text {auto noise }}\right)$ and the mean responses $\left(r_{\text {auto_signal }}\right)$ are shown for the window sizes of 5, 10, 25, 50, 100, and $200 \mathrm{~ms}$. With respect to the sequential correlation of the noise: Dip/Trend: the delays between the sample windows up to which $r_{\text {auto noise }}$ in the onset period is significantly below $(P<0.05)$ or shows a trend $(P<0.1)$ to be below both

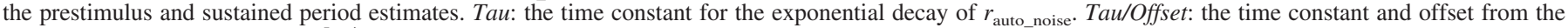
regression $r_{\text {sequ }}=$ Offset $+\beta_{1} e^{L a g / \tau}$. With respect to the sequential correlation of the signal: Tau: the time constant for the exponential decay of $r_{\text {auto_signal. }}$. Tau/Offset: the time constant and offset from the regression $r_{\text {sequ }}=$ Offset $+\beta_{1} e^{\text {Lag/ } \tau}$. * Significant at $P<0.05$. 
ms). Including a constant offset in the regression (i.e., fitting $r_{\text {sequ }}=$ Offset $\left.+\beta_{1} e^{L a g / \tau}\right)$ showed significant $(P<0.05)$ offset for the prestimulus data $(0.015 \pm 0.002$ using 100 -ms windows, Fig. $6 B$ ), significant offsets being observed in the prestimulus period for window sizes down to $5 \mathrm{~ms}$ (Table 1). The absence of evidence for a long-term offset of $r_{\text {auto_noise }}$ in the sustained period is attributable to the decorrelation (the dip in $\left.r_{\text {autonnoise }}\right)$ at onset. Specifically, the sustained period of the responses is taken from 300 to 400 poststimulus onset. At a lag of $200 \mathrm{~ms}$, the two $100-\mathrm{ms}$ windows are $(0 \ldots 100)$ and (300. . 400). The first of these windows falls before the typical response latency (70-100 ms) of STSa neurons (Kiani et al. 2005; Oram and Perrett 1992, 1996), precisely the period where decorrelation occurs (see Figs. 1, 3, and 4).

The exponential of the decay in $r_{\text {auto_noise }}$ with lag between the samples across multiple window sizes indicates the presence of multiple short timescale correlations present in the spike-generating process. As with MT (Bair et al. 2001) and V1 (Kohn and Smith 2005), the correlation of the trial-by-trial variability within the responses of single STSa neurons can last over prolonged periods (indicated by significant offset values). However, the present data suggest the prolonged correlation is disrupted by the presentation of a stimulus even though such correlation can last across many stimulus presentations (Bair et al. 2001).

The sequential correlation of the signal $\left(r_{\text {auto_signal }}\right)$ is also sensitive to the delay between the samples (Fig. $6, B$ and $C$, Table 1). Although the "dip" in $r_{\text {auto_noise }}$ is locked to the onset of the response (Fig. 6A), the rise in $r_{\text {auto_signal }}$ is found when both windows are within the stimulus elicited period of the response (Fig. $6 \mathrm{C}$ ). The requirement for both sample windows to be in the stimulus-elicited response explains 1 ) the decreasing time constant of the decline in $r_{\text {auto_signal }}$ with increasing window size (Table 1) and 2) the accelerating slope of $r_{\text {auto_sig- }}$ nal with increasing lag. For example, the sustained period of the responses is taken from 300 to 400 poststimulus onset. At a lag of $150 \mathrm{~ms}$, the two 50-ms windows (100-150) and (300-350) both fall within the period of stimulus-elicited activity. At a lag of $150 \mathrm{~ms}$, the first of the $100-\mathrm{ms}$ windows $(50 \ldots 150)$ falls partially outside the stimulus-elicited response, the effect being even greater for the $200-\mathrm{ms}$ window $(-50 \ldots 150)$. Longer sample windows at longer lags therefore include data from periods that are not directly driven by the stimulus, leading to a decline of $r_{\text {auto_signal }}$ with increasing slope.

In summary, the low variability (Fano factor) (Amarasingham et al. 2006; Churchland et al. 2006; Oram et al. 2007; Osborne et al. 2004; Uka and DeAngelis 2003) and autocorrelation of the trial-by-trial variability during the initial response (Oram et al. 2007) reflect a transient dip from prestimulus levels. This dip is seen even when the stimulus elicits no response, suggesting a network property rather than mechanisms intrinsic to the neuronal activity per se. The magnitude of the autocorrelation decays exponentially with delay between sample windows, indicating that the correlation is due to processes operating at multiple timescales. As with crosscorrelation in areas MT and V1 (Bair et al. 2001; Kohn and Smith 2005; Smith and Kohn 2008), the autocorrelation seems to last for extended periods. However, the present study indicates that the long-lasting sequential correlation in STSa is transiently disrupted by the presentation of a visual stimulus.

\section{A dual-process model captures the basic statistics of single neuronal responses}

A drop in the Fano factor is expected when activity, assessed using short-duration bins with no more than one spike in, increases. Short-duration bins can be considered as binomially distributed with probability $p$ of a spike in a given millisecond bin. Such bins have mean $p$, variance $p(1-p)$, and thus a Fano factor of $(1-p)$. It follows that as the firing rate $(p)$ increases, the Fano factor $(1-p)$ decreases. A series of such binomial bins, as can be used to model a spike train, will have mean and variance given by the sum of the mean and variances of the individual bins if and only if the bins are independent. Thus spike count distributions assessed over an extended time will have a Fano factor of about 1 only if the bins are independent. Because the Fano factor is $>1$, the bins (activity) must be correlated in time (Kass and Ventura 2006; Oram et al. 1999, 2001). Second, spike count distributions are truncated at 0 , implying that estimates of correlation between two spike counts will change as a function of the mean (de la Rocha et al. 2007). It is thus natural to ask: 1) are the high levels of $r_{\text {auto } \_ \text {noise }}$ in the prestimulus and late response periods due to the Fano factor being $>1$ ? and 2) is the decrease in $r_{\text {auto_noise }}$ due to the rapid change in mean firing rates around response onset?

Artificial spike trains were generated using the binomial approximation of a time-varying (nonhomogeneous) Poisson (NHPP) process. Because the time bins in the NHPP model are independent, the sequential correlation should be 0 . The spike count matched (SCM) model forces the Fano factor of extended windows (here -300 to 600 poststimulus onset) to match that seen in the data (Oram 2005; Oram et al. 1999, 2001, 2002). Thus the SCM model was used to investigate the impact of a Fano factor $>1$ on the autocorrelation measures $r_{\text {auto noise }}$ A drop in autocorrelation of the trial-by-trial variability is also indicative of a change in the (spike-) generating process (Kohn and Smith 2005). The SCM model was therefore run as two independent processes with a change in process at $70 \mathrm{~ms}$ (SCM2 model; see METHODS for details).

The sequential correlation ( $r_{\text {auto_noise }}$ and $\left.r_{\text {auto_signal }}\right)$ and the Fano factor using sample window sizes ranging from 5 to 200 $\mathrm{ms}$ of the three spike train models are shown in Fig. 7. As 57 expected, the binomial approximation of the nonhomogeneous Poisson process (NHPP, left) gives a Fano factor slightly $<1$ (Fig. 7A) and zero sequential correlation $r_{\text {auto noise }}$ (Fig. 7D), irrespective of sample window size. The SCM and SCM2 models (Fig. 7, middle and right columns, respectively) have a Fano factor $>1$ (Fig. 7, $B$ and $C$ ) that, as described earlier, induces nonzero $r_{\text {auto noise }}$ (Kass and Ventura 2006; Oram et al. 1999, 2001; also see Fig. 7, $E$ and $F$ ). Consistent with the neurophysiological data, the SCM and SCM2 models show an increase of both Fano factor and $r_{\text {auto_noise }}$ with increasing window size.

The dips in Fano factor and $r_{\text {auto noise }}$ around response onset are absent in the NHPP and SCM simulated spike trains. The dips seen in the neurophysiological data, however, are captured by the SCM2 model (Fig. 7, $C$ and $F$ ). The transient changes shown by the SCM2 model also occur without change in firing rate (data not shown). Furthermore, the Fano factor and $r_{\text {auto noise }}$ of the SCM2 model data are, like the neurophysiological data, comparable between the "prestimulus" and "sustained" periods (Fig. 7, $C$ and $F$ ), despite the difference in 

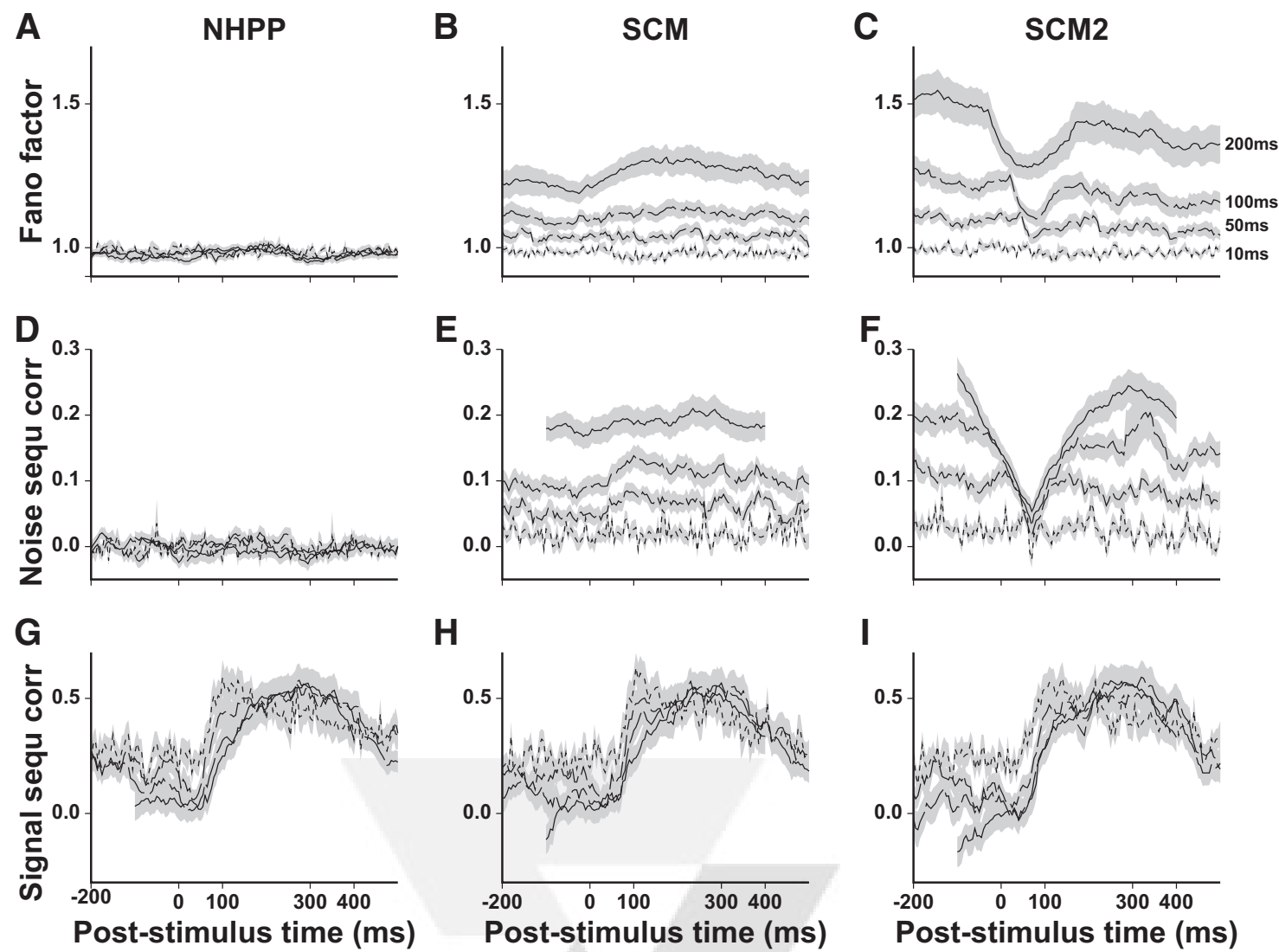

FIG. 7. Modeling statistics of single neurons. The Fano factor $(A-C)$, the sequential correlation of the noise $\left(r_{\text {auto noise }}, D-F\right)$, and sequential correlation of the signal ( $r_{\text {auto signal }}, G-I$ ) of the nonhomogeneous Poisson process (NHPP, left column), the spike count matched model (SCM, middle column), and dual-process spike count matched model (SCM2, right column) are shown. Values were calculated using windows of 10, 50, 100, and 200 ms (solid, short-dash, dashed, long-dash, solid lines, respectively). Shaded areas represent \pm SE.

firing rate. The similarity of $r_{\text {auto_signal }}$ across the three models (Fig. 7, $G-I$ ) is expected because the same spike density functions (mean activities) are used for all three models.

Variations of the Fano factor and $r_{\text {auto noise }}$ with window size and lag in the different spike train models are shown in Fig. 8. The ability of the different models to explain the neurophysiological results was assessed using the correlation coefficient

T2,AQ:2 between model and neuronal data (Table 2). The absence of modulation over time in the Fano factor or the $r_{\text {auto_noise }}$ with either window size or lag of the NHPP model data (Fig. 8, $A$, $D$, and $G$ ) is reflected in the poor fits to the neuronal data, both overall and for the individual window size, with a clear tendency toward negative correlation (Table 2). Although the SCM model captures some of the dependence of Fano factor and $r_{\text {auto noise }}$ on window size and delay between samples (overall $R^{2}=0.81$ and 0.29 , respectively), the values are consistent across prestimulus, onset, and sustained response periods. The SCM2 model captures the dip in the Fano factor and $r_{\text {auto noise }}$ around response onset and therefore provides a better description overall (Fano factor: $R^{2}=0.96 ; r_{\text {auto noise }}$ : $\left.R^{2}=0.64\right)$ and for window sizes $>10 \mathrm{~ms}$ (Table 2 ) than the other models.

In summary, comparison of the results from the NHPP model with the results from the SCM and SCM2 models indicates that observed high levels of $r_{\text {auto noise }}$ in the prestimulus and late response periods are largely attributable to the Fano factor being $>1$. The dip around response onset of Fano factor and sequential correlation is not seen in the NHPP and
SCM models and thus cannot be attributed to the change in firing rate at response onset nor bias due to limited sample size (see METHODS). Although the SCM2 model captures much of the "shape" of the response statistics, it is not a complete description. The SCM2 model predicts lower than observed values of the sequential correlation found using small windows (e.g., $40 \%$ of the observed values using 5-ms windows) and overestimates the values for large windows (e.g., $136 \%$ of the observed values using 200-ms windows). This mismatch suggests the SCM2 model is too simple in that it does not include multiple timescales. However, it is noteworthy that the mismatch in sequential correlation between observed and model values is smaller for the SCM2 model than that for either of the other models.

\section{Response statistics between neurons}

The average signal and noise correlations of 67 pairs of neurons simultaneously recorded from STSa are shown for different window sizes as a function of time in Fig. 9. As F9 expected (Averbeck and Lee 2003; Kass and Ventura 2006; Reich et al. 2001a), the correlation of the trial-by-trial variability ("Noise" correlation) between neurons and the correlation of the mean (signal) between neurons increased with increasing window size (Fig. 9B: $r_{\text {cross noise }}$; Fig. 8D: $\left.r_{\text {cross_signal }}\right)$. Despite the variation in the magnitude of the noise correlation $r_{\text {cross_noise, }}$ the dip is detectable even with a 5-ms sample window. The reduction in the cross-correlation of the 

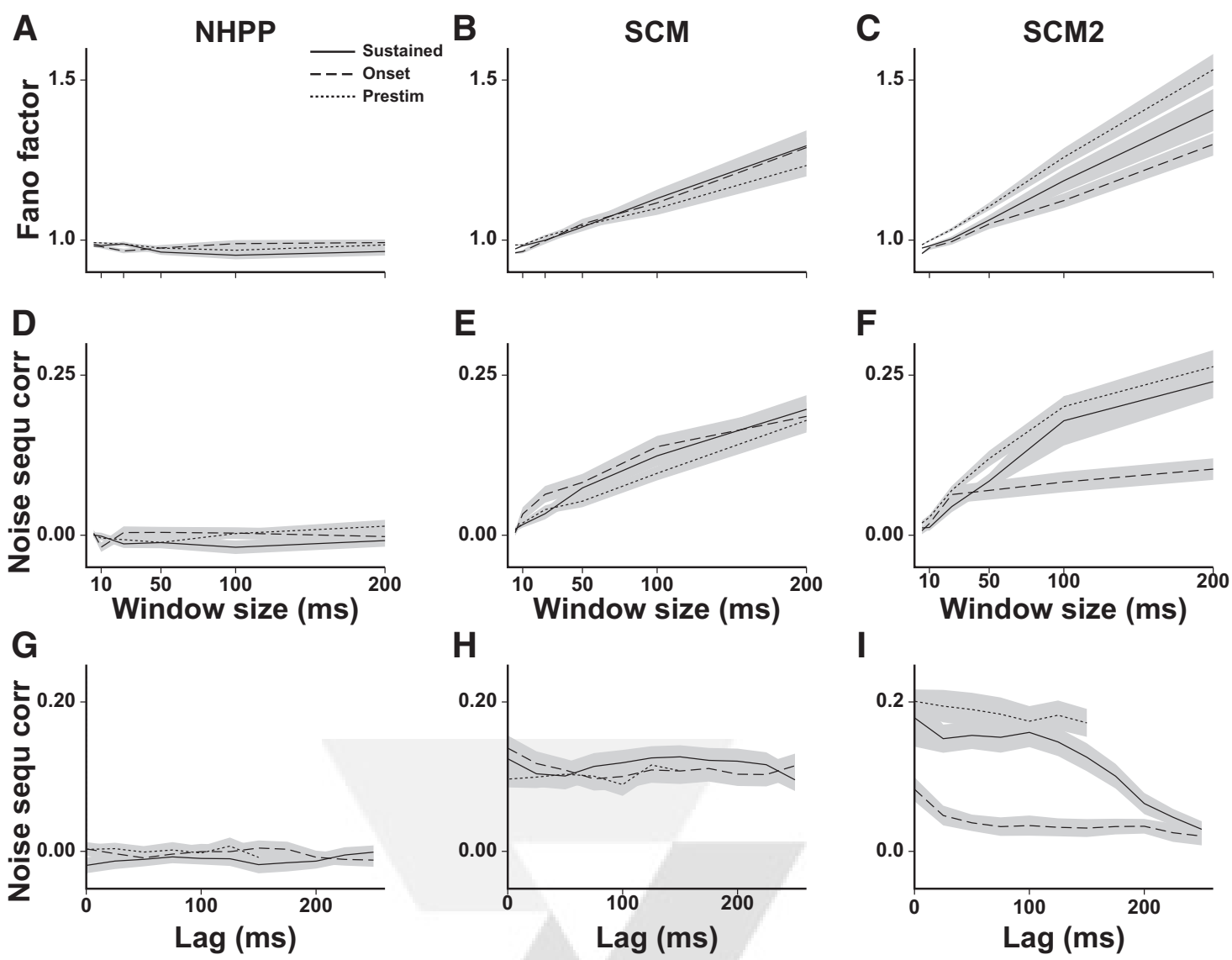

FIG. 8. Dependence of modeled statistics on window size and lag. The mean Fano factor $(A-C)$, the sequential correlation of the noise $\left(r_{\text {auto }}\right.$ noise,$\left.D-F\right)$ as a function of window size, and $r_{\text {auto noise }}$ as a function of lag between samples $(G-I)$ of the nonhomogeneous Poisson process (NHPP, left column), the spike count matched model (SCM, middle column), and dual-process spike count matched model (SCM2, right column). Shaded areas represent \pm SE. Values were calculated using independent samples in the periods $-200 \ldots .0 \mathrm{~ms}$ (Prestim, dotted lines), 70. .120 ms (Onset, dashed lines), and 300. . .400 ms (Sustained, solid lines) poststimulus onset.

noise is transitory, returning to levels comparable to the prestimulus period at roughly $150-200 \mathrm{~ms}$ poststimulus onset. The signal correlation shows a slight dip after response onset but remains high until after the response itself has stopped ( $\sim 450 \mathrm{~ms}$; see Fig. 3).

Taking the 100-ms sample window as an example, the correlation of the trial-by-trial variability across neurons (Fig. 9$, top $)$ is relatively high in the prestimulus period $\left(r_{\text {cross_noise }}=\right.$ $0.28 \pm 0.02)$ and equivalent $(P=0.1)$ during the sustained period of the response $(0.24 \pm 0.02)$. The noise correlation at response onset $(0.18 \pm 0.02)$ is significantly less than that at either prestimulus or sustained periods of the response $(P<$ 0.005 , each comparison). The values of $r_{\text {cross noise }}$ in the sustained period using sample windows of $\geq 100 \mathrm{~ms}$ are typical of cortical visual areas V1 (Gawne et al. 1996; Kohn and Smith 2005; Reich et al. 2001b), MT (Bair et al. 2001; Zohary et al. 1994), and IT (Gawne and Richmond 1993). However, the correlation of the trial-by-trial variability between neurons falls significantly below the prestimulus level $40 \mathrm{~ms}$ after stimulus onset, reaching a minimum of $0.16 \pm 0.02$ some $75 \mathrm{~ms}$ poststimulus onset. The $r_{\text {cross_noise }}$ then rises, being statistically indistinguishable $(P>0.05)$ from prestimulus levels $175 \mathrm{~ms}$ after stimulus onset.

Trivially, the signal correlation between neurons rises around response onset (Fig. 9C). Although $r_{\text {cross_signal }}$ increases with increasing window size, it plateaus at window sizes of $\geq 50 \mathrm{~ms}$ (Fig. 9D). The $r_{\text {cross signal }}$ at response onset $(0.80 \pm$ 0.10 with a 100 -ms window) and in the period $300-400 \mathrm{~ms}$ $(0.81 \pm 0.08)$ is comparable with, albeit slightly less than, previous studies examining signal correlation of visually responsive neurons recorded from single electrodes (e.g., 0.86 in MT; Bair et al. 2001).

The signal correlation is nonzero in the prestimulus period (e.g., with 100 -ms window, $r_{\text {cross_signal }}=0.31 \pm 0.04$ in the $200 \mathrm{~ms}$ before stimulus onset), indicating the mean activity level in the prestimulus period varied with the subsequent stimulus. The neuronal response to one stimulus influences responses to a following stimulus in visual (Dragoi et al. 2002; Felsen et al. 2002; Grill-Spector et al. 2006; Kourtzi and Kanwisher 2001; Macknik and Livingstone 1998; Sawamura et al. 2006), auditory (Wehr and Zador 2005), and somatosensory systems (Khatri et al. 2004), even with intervening stimuli (Felsen et al. 2002; Perrett et al. 2009). Although stimuli were presented in random order, the presentations were blocked such that each stimulus was presented once before any individual stimulus was repeated. Because the stimuli were specifically selected to be either effective or ineffective, the presentation of an effective stimulus is likely to have been preceded by an ineffective stimulus and vice versa. Thus interaction between stimulus presentations (Perrett et al. 2009) would produce this initially unexpected observation. 
TABLE 2. Correlation of model and neuronal data

\begin{tabular}{|c|c|c|c|}
\hline Variable & NHPP & SCM & $\mathrm{SCM} 2$ \\
\hline \multicolumn{4}{|c|}{ A. Fano factor } \\
\hline Overall & -0.35 & $0.90 * *$ & $0.98^{* *}$ \\
\hline \multicolumn{4}{|c|}{ B. $r_{\text {auto_noise }}$} \\
\hline Zero lag & -0.32 & 0.43 & 0.77 \\
\hline Overall & $-0.33 * *$ & $0.54 * *$ & $0.80^{* * *}$ \\
\hline $5 \mathrm{~ms}$ & 0.09 & 0.29 & 0.21 \\
\hline $10 \mathrm{~ms}$ & $-0.36^{*}$ & 0.26 & 0.14 \\
\hline $25 \mathrm{~ms}$ & $-0.42^{*}$ & 0.28 & $0.65^{* *}$ \\
\hline $50 \mathrm{~ms}$ & -0.22 & 0.03 & $0.75^{* *}$ \\
\hline $100 \mathrm{~ms}$ & -0.22 & -0.04 & $0.77 * *$ \\
\hline $200 \mathrm{~ms}$ & -0.37 & $0.63 * *$ & $0.90 * *$ \\
\hline \multicolumn{4}{|c|}{ C. $r_{\text {auto_signal }}$} \\
\hline Overall & $0.40^{* *}$ & $0.34 * *$ & $0.31 * *$ \\
\hline $5 \mathrm{~ms}$ & $0.53 * *$ & $0.49 * *$ & $0.48^{* *}$ \\
\hline $10 \mathrm{~ms}$ & $0.79 * *$ & $0.78^{* *}$ & $0.79 * *$ \\
\hline $25 \mathrm{~ms}$ & $0.60 * *$ & $0.57 * *$ & $0.57 * *$ \\
\hline $50 \mathrm{~ms}$ & -0.15 & -0.25 & -0.27 \\
\hline $100 \mathrm{~ms}$ & 0.29 & 0.25 & 0.17 \\
\hline $200 \mathrm{~ms}$ & $0.57 * *$ & $0.45^{*}$ & $0.44 *$ \\
\hline
\end{tabular}

The correlation coefficient between the neural data and the different models (NHPP, nonhomogeneous Poisson process; SCM, spike count matched model; SCM2, dual-process spike count matched model) of the Fano factor, the sequential correlation of the trial-by-trial variability ( $\left.r_{\text {autonnoise }}\right)$ and the sequential correlation of the mean response $\left(r_{\text {auto_signal }}\right)$. * Significant at $P<0.05$; **significant at $P<0.05$.

The impact of the lag between sample windows on $r_{\text {cross_noise }}$ and $r_{\text {cross signal }}$ is shown in Fig. 10. The cross-correlation of the

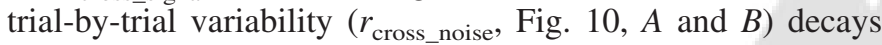
smoothly as a function of the lag between the sample windows. The cross-correlation of the signal ( $r_{\text {cross signal }}$, Fig. 10, $C$ and $D$ ) with increasing lag also decays smoothly in the prestimulus and sustained periods of the response (Fig. 10D, dotted and solid lines, respectively). However, there is a marked "step" in $r_{\text {cross signal }}$ with lags around response onset (Fig. 10D, dashed line), with a rapid decline from lags of 50 to $75 \mathrm{~ms}$. Indeed, at lags of $25 \mathrm{~ms}$ (not shown) and $50 \mathrm{~ms}$ (Fig. 10C, dashed line) there is a decrease in $r_{\text {cross_signal }}$ around 50-70 ms that is not captured in the 70 - to $120-\mathrm{ms}$ onset period. Thus the signal at response onset of a neuron is relatively uncorrelated with the previous activity of other neurons.

\section{SCM2 model captures the basic statistics of responses between neuronal pairs}

The SCM2 model also captures the transient reduction (dip) in the cross-correlation of the between-neuron trial-by-trial variability. A dip in the cross-correlation of the SCM2 modeled data is evident for all window sizes (Fig. 11, top) at the time F11 when a change of process $(70 \mathrm{~ms})$ occurs. The dip in $r_{\text {cross_noise }}$ is not seen using either the NHPP or slightly modified SCM models (not shown). The presence of the dip shows that when sample windows span the time at which a change in process occurs, a change of process in the individual neurons induces detection of a drop in the cross-correlation of the trial-by-trial variability between neurons.

As with the responses of single neurons, the SCM2 model does not provide a full description of the neurophysiological data, despite capturing the main features. The absence of decay with lag in the SCM2 modeled data (Fig. 11, bottom) is to be expected simply because a single, constant process is used in the interval $[-300 \ldots 70 \mathrm{~ms}]$ and a second process in the interval [71. .600 ms]. The SCM2 models predict substantially lower than observed values of $r_{\text {cross-noise }}$ for all window sizes examined, the mismatch being proportionally smaller for large windows $\left(r_{\text {cross_noise }}: 200\right.$-ms sample window, SCM2 $\approx$ 0.22 , $\mathrm{STSa} \approx 0.30 ; 10$-ms sample window $\mathrm{SCM} 2 \approx 0.02$, $\mathrm{STSa} \approx 0.15$ ).

\section{Implications for neuronal coding}

The drop in response variability and the drop in crosscorrelation of the trial-by-trial variability associated with response onset have implications for the information that the neuronal responses carry. Specifically, I ask what is the impact of the transient dip in the Fano factor and $r_{\text {cross_noise }}$ on the mutual information between the responses and the input stimuli? To calculate the mutual information between a stimulus set and the responses of a pair of model neurons, the mean response of each neuron to each stimulus needs to be known, as does the trial-by-trial variability of the responses about that
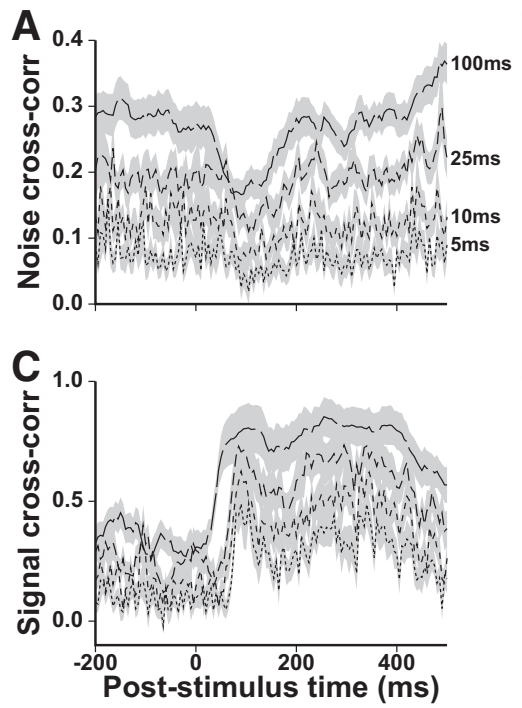

B
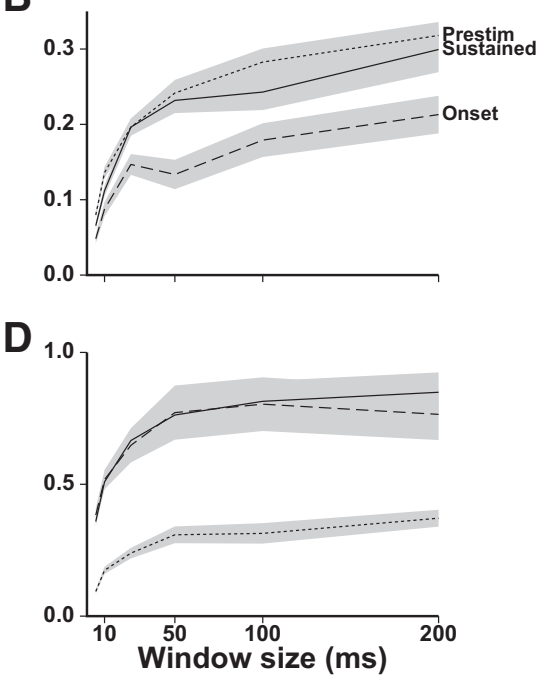

FIG. 9. Correlation between pairs of neurons. The mean cross-correlation $( \pm \mathrm{SE})$ of the noise $\left(r_{\text {cross_noise }}, A\right.$ and $\left.B\right)$ and signal $(C$ and $D$ ) from pairs of simultaneously recorded neurons. $A$ and $C$ show the cross-correlation for windows of 5,10 , 25, 50, 100, and $200 \mathrm{~ms}$ (dotted, short-dash, dashed, long-dash, vs. long dash, solid lines, respectively). Right panels show the mean cross-correlation of the noise $(B)$ and signal $(D)$ in the prestimulus ( -200 to $0 \mathrm{~ms}$ poststimulus onset, dotted line) period, during response onset $(70-120 \mathrm{~ms}$ poststimulus onset, dashed line), and in the sustained period of the response (300-400 ms poststimulus onset, solid line) as a function of the sample window size. 

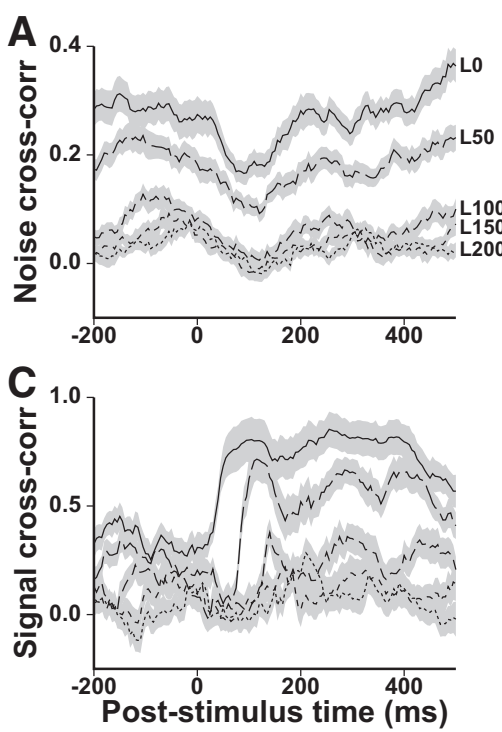

B
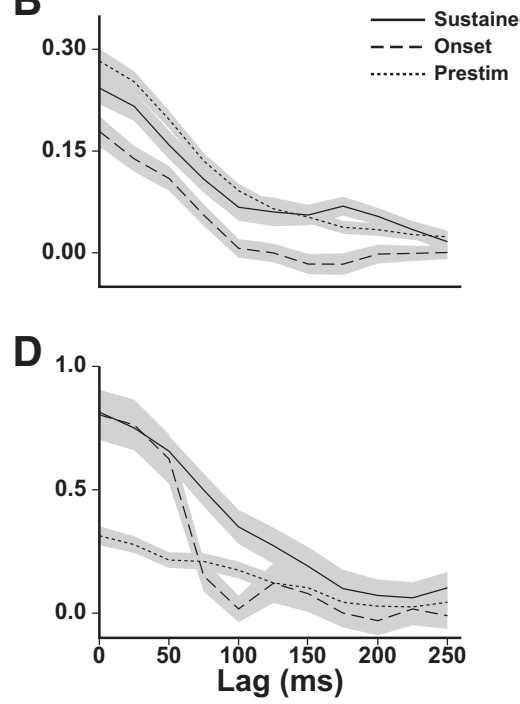

FIG. 10. Correlation between pairs of neurons as a function of lag between samples. The mean cross-correlation $( \pm \mathrm{SE})$ of the noise $\left(r_{\text {cross_noise }}, A\right.$ and $\left.B\right)$ and signal $\left(r_{\text {cross_signal }}, C\right.$ and $\left.D\right)$ from pairs of simultaneously recorded neurons. $A$ and $C$ show the cross-correlation for 100-ms windows with a lag or delay of $0,50,100,150$, and $200 \mathrm{~ms}$ (solid, long-dash, dash, short-dash, dotted lines, respectively). Right panels show the mean crosscorrelation of the noise $(B)$ and signal $(D)$ in the prestimulus ( -200 to $0 \mathrm{~ms}$ poststimulus onset, dotted) period, during response onset (70-120 ms poststimulus onset, dashed), and in the sustained period of the response (300-400 ms poststimulus onset, solid) as a function of the lag between the sample windows. mean (Fano factor). It is also necessary to know the covariance structures between the neurons, both of the signal $\left(r_{\text {cross_signal }}\right)$ and the trial-by-trial variability ( $\left.r_{\text {cross_noise }}\right)$. Thus the information needs to be calculated twice: once with the low Fano factor and $r_{\text {cross noise }}$ observed at response onset and once with the higher values observed in both the prestimulus and sustained sections of the activity. Because the dip in Fano factor and $r_{\text {cross_noise }}$ occurs at the time of response onset when there is a large transient peak in firing rate, comparison of the mutual
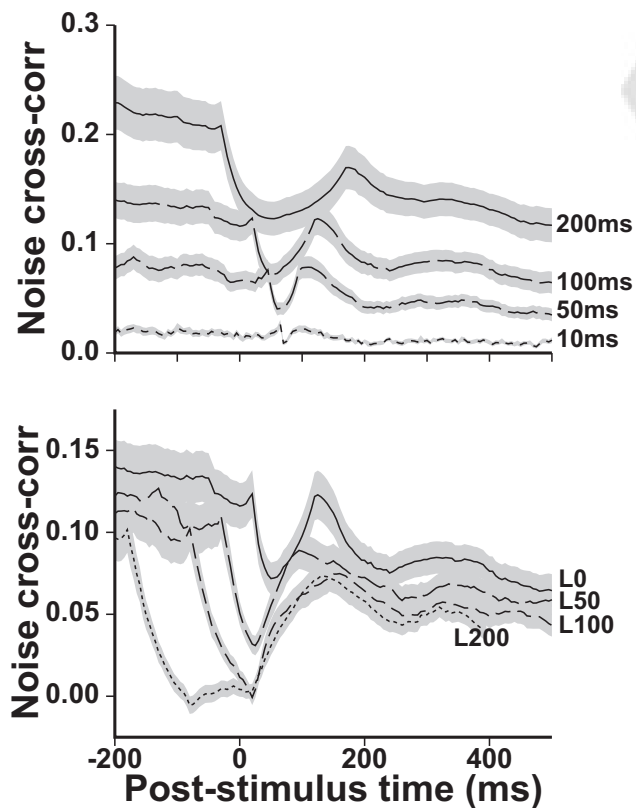

FIG. 11. SCM2 model predicts dip in cross-correlation. The mean crosscorrelation $( \pm \mathrm{SE})$ of the noise $\left(r_{\text {cross_noise }}\right)$ from the SCM2 model. The cross-correlation was calculated using 100 surrogate trials for every trial in the neurophysiological data. Top: the cross-correlation of the noise assessed using windows of 10, 50, 100, and $200 \mathrm{~ms}$ are shown (short-dash, dash, long-dash, and solid lines, respectively). A dip in the cross-correlation is evident for all window sizes at the time when a change of process $(70 \mathrm{~ms})$ occurs. Bottom: the cross-correlation of the noise assessed using a 100-ms window with lags of 0 , 50, 100, and $200 \mathrm{~ms}$ are shown (solid, long-dash, dash, short-dash, and dotted lines, respectively). Although the change of process in the SCM2 model causes decorrelation (the dip), the correlation, unlike the neural data, does not decay with time. information during the onset and sustained periods would confound changes in mean response with changes in the variability and correlation structure. Therefore it is necessary to model the impact of the transient dip in the Fano factor and $r_{\text {cross noise }}$ on the mutual information.

The distribution of the signal (mean response to different stimuli) was modeled by setting each neuron to have the average tuning curve found in area STS to changes in a perspective view of the head (Oram 2005; Perrett et al. 1991). The relative "position" of the tuning curves between the pair of model neurons was adjusted to give the observed signal correlation $\left(r_{\text {cross_signal }}\right)$. The mutual information was calculated using 128 stimuli, the mean firing rate (signal) to each stimulus given by the tuning curve. Because the stimuli were uniformly distributed across the modeled stimulus space (perspective view), the tuning curve ensuring the distribution within "response space" is typical of STSa neurons. The peak response of the tuning curve was taken as the response to the effective stimuli at $100 \mathrm{~ms}$ poststimulus onset (Fig. 5, inset), scaled by the sample window $(10,25,50,100$, or $200 \mathrm{~ms})$. The mutual information between the pair of modeled responses and 128 stimuli was assessed using the average Fano factor, signal correlation ( $\left.r_{\text {cross_signal }}\right)$, and cross-correlation of the noise ( $\left.r_{\text {cross noise }}\right)$ observed at $100 \mathrm{~ms}$ poststimulus onset (Fig. 12, F12 solid line). As can be seen, the information increases with the integration time (window size), reaching $95 \%$ of the maximum with a window size of $50 \mathrm{~ms}$ (see also Gershon et al. 1998). The mutual information in the absence of the transient dip of the Fano factor and cross-correlation of the noise was assessed using the average Fano factor and cross-correlation of the noise from the prestimulus period while maintaining the same distribution of mean responses (signal) and signal correlation (Fig. 12, dashed line).

The increase in the mutual information between the stimuli and pairs of neuronal responses due to the transient dips in the Fano factor and $r_{\text {cross_noise }}$ is evidenced as the difference between the solid and dashed lines of Fig. 12. The increase was minimal for small windows (10-ms sample window; the increase in the mutual information was only $1 \% ; 25 \mathrm{~ms}$, increase of $7 \%$ ). The dips in Fano factor and $r_{\text {cross_noise }}$ around response onset found with windows of 50, 100, and $200 \mathrm{~ms}$ resulted in 
13,19 , and $24 \%$ increases in mutual information respectively compared with the mutual information when the Fano factor and $r_{\text {cross noise }}$ were set to the values observed in the prestimulus periods. Given the similarity between the prestimulus and sustained periods of the response with respect to the Fano

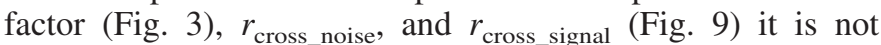
surprising than almost identical results were obtained using the values from the sustained period of the response $(300-400$ $\mathrm{ms})$. Thus the transient dip in Fano factor and cross-correlation of the noise described here can result in appreciable changes in the efficiency of the neural coding, particularly when the integration time is $\geq 100 \mathrm{~ms}$.

\section{I S C U S S I O N}

The response statistics of single and pairs of visually responsive neurons in the anterior sections of the superior temporal cortex were examined. Because the trial-by-trial variability of neuronal responses is proportional to the mean firing rate, the normalized variability (variance/mean) was used to examine changes in response variability. The data indicate that the normalized variability is high in the prestimulus period (i.e., in the absence of a stimulus) and roughly equivalent to that in the sustained period of the response. There is a dip, however, in normalized variability of the initial visually evoked response in STSa, as seen in V1 (Muller et al. 2001) and in previous studies of inferior temporal cortex and STSa (Amarasingham et al. 2006; Oram and Perrett 1992; Vogels and Orban 1991). Variability is reduced for high response magnitudes (Hartveit and Heggelund 1994). However, the drop in Fano factor is seen with stimuli that elicit negligible responses (Fig. 5) and is absent in both the NHPP and SCM models (Fig. 7), which include the relevant changes in activity over time. Thus changes in activity at response onset cannot explain the observed changes in response variability. The fixation window used here was quite large ( $6^{\circ}$ across). Previous studies have noted that a considerable proportion of trial-by-trial response variability in V1 and LGN can be attributed to small variations in eye position (Gur et al. 1997). As already noted, the values for the normalized variability (Fano factor) and autocorrelation $\left(r_{\text {auto noise }}\right)$ are almost identical to those obtained in previous

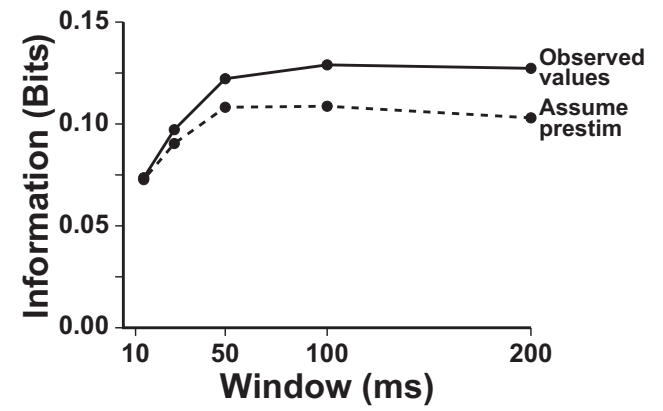

FIG. 12. Mutual information carried by pairs of neurons. The mutual information between pairs of neurons for the response magnitudes at $100 \mathrm{~ms}$ poststimulus onset is plotted as a function of sample window size. The information using the observed values of the average of the Fano factor of the individual neurons and $r_{\text {cross_noise }}$, the trial-by-trial cross-correlation (solid line) is higher than using the average values from the prestimulus period $(-200 \ldots$. .0, dashed line). The signal correlation between the pairs of neurons was fixed at the value observed at $100 \mathrm{~ms}$ poststimulus onset. The information when the Fano factor and $r_{\text {cross_noise }}$ were taken from the sustained period was almost identical to that taken from the prestimulus period. studies with much tighter eye movement control (e.g., Bair 1999), as is the presence of the "dip" around response onset (e.g., Amarasingham et al. 2006; Churchland et al. 2006). Furthermore, the response variability of IT neurons is relatively independent of the amount of eye movement so long as the stimulus remains well within the receptive field (Wiener et al. 2001). Thus although the lack of precise eye-movement control may have introduced small errors in the absolute values of response variability, the impact of this on the normalized response statistics used here is likely to be negligible.

As with response variability, the response autocorrelation has a marked "dip" in the first $100 \mathrm{~ms}$ of the stimulus-elicited response. The transient reductions in response variability and autocorrelation are also seen in the responses to ineffective stimuli (Fig. 5), indicative that the effects are the results of a network property rather than intracellular activity-dependent mechanisms. The autocorrelation decayed in an exponential fashion with increasing lag between the two sample windows (Fig. 6), the effect being seen at multiple timescales (sample windows of 10 to $200 \mathrm{~ms}$, Table 1). It is also noteworthy that the sequential correlation does not decay to 0 , instead falling to some low value $(\sim 0.02)$. Thus like others (Bair et al. 2001), we find evidence for multiple timescales, including long-term correlation of neuronal activity.

Comparison of the NHPP and SCM models (Fig. 7) with the neuronal data shows that the correlation induced by the nonPoisson distribution of spike count (Kass and Ventura 2006; Oram et al. 1999) is insufficient to explain the levels of correlation in the data. Previous studies have shown that a dip in cross-correlation between neurons can result from a change in response statistics (Poisson to gamma processes) without a change in firing rate (Tetzlaff et al. 2008). The present simulations suggest that the "dip" in response variability and autocorrelation of single neurons around response onset can be induced simply by a change of process, without any change in the underlying output statistics, and that such changes are reflected in the between-neuron correlation.

Perhaps the simplest mechanism that could underlie a change in the spike-generation process at response onset involves a change in the relative importance of feedforward inputs. The number of feedforward synapses to layer $4 \mathrm{~V} 1$ neurons of the cat is estimated to be only about $2 \%$ of the total (Binzegger et al. 2004; Douglas and Martin 2007), whereas roughly $12 \%$ of synapses to LGN come from retinal inputs (Van Horn et al. 2000). Although small in number, these feedforward connections can clearly drive the target neurons. However, the relative contribution of feedforward connections during undriven (prestimulus) activity is likely to be substantially less than that in the driven state. In the absence of driving input, the neuronal activity will be determined largely by feedback and lateral connections. When receiving sensory inputs the balance might change, such that the activity driving the spike-generation process is now governed by the small proportion of active feedforward inputs. Over time the relative importance of recurrent activity will rise as the stimuluselicited activity spreads through the network and activates the recurrent connections. The transient change in setting the neuron's activity from recurrent to feedforward inputs and then back to recurrent inputs would provide the change of generating process suggested by the SCM2 model. 
Recent studies indicate that mechanisms other than a change in process may also play a role in the dip of response variability and correlation at response onset. Variability of responses in LGN, as measured by the Fano factor, is reduced by cortical feedback from V1 (Andolina et al. 2007). The transient dip in Fano factor and autocorrelation reported here and elsewhere could therefore be due to increased feedback. However, feedback to LGN is dominated from extraarea (V1) input, whereas the majority of recurrent connections in cortex are within the cortical area (Binzegger et al. 2004; Douglas and Martin 2007). It remains to be seen whether the dips in Fano factor, autocorrelation, and cross-correlation in STSa neural responses are still seen in the absence of feedback from other cortical areas.

The trial-by-trial variability between neurons becomes less correlated for about $100 \mathrm{~ms}$ starting at response onset (Smith and Kohn 2008), at the same time as the signal correlation increases (Fig. 9, $C$ and $D$ ). The dip in noise correlation is concurrent with the increase in signal correlation, suggesting that the "noise" from one input neuron is independent of other neurons, even when those other neurons convey related information. The overall impact of the transient dips in response variability of individual neurons and the covariance of the trial-by-trial variability between neurons is to increase the information conveyed by pairs of neurons by $\leq 25 \%$.

Whenever neurons are recorded on the same electrode, there is always a concern that incomplete or inaccurate clustering will lead to artifactually high measures of cross-correlation. Considerable effort was made to ameliorate this possibility in the spike-sorting stage by using a high threshold for cluster separation (see METHODS) and through visual examination of the spike waveforms. It is also noteworthy that the cross-correlation coefficients reported here are toward the lower end of those reported in other studies using spikes sorted from single neurons (e.g., Bair et al. 2001) and where multiple electrodes were used (e.g., Kohn and Smith 2005; Smith and Kohn 2008). The data presented here are consistent with previous reports and, if anything, suggest lower rather than higher correlation than that of previous studies, suggesting that the potential confounds are small. Furthermore, the focus here has been in the "dip" in the correlation rather than the absolute magnitude and it is highly unlikely that this is a result of cross-contamination between clusters.

The mean firing rates of neurons were found to be correlated in the prestimulus period (Fig. 9, $C$ and $D$ ), an effect probably reflecting the limited number of stimuli (typically $8-20$ ) and interactions between stimuli. The ongoing activity related to previous stimuli, however, is independent of subsequent stimulus-elicited activity (Fig. 10, C and D) and is therefore probably different from those mechanisms underlying similar effects associated with attention (Reynolds and Chelazzi 2004). The SCM 2 model shows that this effect may, like the dips of the within-neuron response statistics, be an artifact of sampling an instantaneous change of process in the individual neurons rather than a change in the between-neuron statistics (Fig. 11). Thus although the correlation between pairs of LGN neurons is reduced by cortical feedback from V1 (Andolina et al. 2007), the present study shows that a change of generating process within the activity of single neurons explains much of the transient changes in the between-cell correlation.

Although the simple SCM2 model shows the transient "dip" in Fano factor, autocorrelation, and cross-correlation of the responses, the model fails to capture the absolute magnitudes and the dependence of the correlation measures on lag. The decay of the correlation measures with lag between the sample windows indicates that the neuronal activity is governed at multiple timescales (Bair et al. 2001). The high level of interconnectivity within (Binzegger et al. 2004; Douglas and Martin 2007; Stratford et al. 1996) and between (Felleman and Van Essen 1991; Young 1993; Young et al. 1994) cortical areas implies that the feedback/lateral inputs will operate through multiple loops of differing lengths and thus show autocorrelation and cross-correlation of their activity across multiple timescales. It remains to be seen whether incorporation of recurrent activity through multiple loops of different length into models of neural activity induce more accurate absolute measures of correlation as well as show the decrease of correlation with lag between sample windows.

In summary, the main neurophysiological findings reported here are of a transient drop in response variability and correlation of the trial-by-trial variability both within and between neurons at response onset. Although these decreases are detectable using sample windows as small as 5-ms duration, the effects are more noticeable with sample windows $\leq 100 \mathrm{~ms}$. Furthermore, the effects are seen in the absence of significant stimulus-induced changes in activity levels. Although the transient changes in response statistics appear relatively complex, modeling indicates that a simple change or resting of underlying process generating the neuronal activity within individual neurons can give rise to the complex observed neurophysiological phenomena. Critically, a change of process within single modeled neuronal spike trains induces changes in the response statistics of those spike trains between neurons. This simple mechanistic account of the transient changes in the response statistics of both single and pairs of neurons may underlie up to a $25 \%$ increase in the information conveyed by a neuronal population in the initial stimulus-induced response.

\section{A C K N OWLEDGMENTS}

I thank the unknown reviewers for helpful and clarifying comments and D. M. Endres and M. C. van Rossum for stimulating discussion and comments on this manuscript.

\section{G R A N T S}

This work was supported by European Union Framework Grant FP5Mirror.

\section{IS C L OS URES}

No conflicts of interest, financial or otherwise, are declared by the author.

\section{REFERENCES}

Abbott LF, Dayan P. The effect of correlated variability on the accuracy of a population code. Neural Comput 11: 91-101, 1999.

Amarasingham A, Chen TL, Geman S, Harrison MT, Sheinberg DL. Spike count reliability and the Poisson hypothesis. J Neurosci 26: 801-809, 2006.

Anderson B, Sanderson MI, Sheinberg DL. Joint decoding of visual stimuli by IT neurons' spike counts is not improved by simultaneous recording. Exp Brain Res 176: 1-11, 2007.

Andolina IM, Jones HE, Wang W, Sillito AM. Corticothalamic feedback enhances stimulus response precision in the visual system. Proc Natl Acad Sci USA 104: 1685-1690, 2007.

Averbeck BB, Lee D. Neural noise and movement-related codes in the macaque supplementary motor area. J Neurosci 23: 7630-7641, 2003. 
Averbeck BB, Lee D. Coding and transmission of information by neural ensembles. Trends Neurosci 27: 225-230, 2004.

Averbeck BB, Lee D. Effects of noise correlations on information encoding and decoding. J Neurophysiol 95: 3633-3644, 2006.

Baddeley R, Abbott LF, Booth MCA, Sengpiel F, Freeman T, Wakeman EA, Rolls ET. Responses of neurons in primary and inferior temporal visual cortices to natural scenes. Proc R Soc Lond B Biol Sci 264: 1775-1783, 1997.

Bair W. Spike timing in the mammalian visual system. Curr Opin Neurobiol 9: 447-453, 1999.

Bair W, Zohary E, Newsome WT. Correlated firing in macaque visual area MT: timescales and relationship to behavior. J Neurosci 21: 1676-1697, 2001.

Barraclough NE, Keith RH, Xiao D, Oram MW, Perrett DI. Visual adaptation to goal-directed hand actions. J Cogn Neurosci 21: 1806-1820, 2009.

Barraclough NE, Xiao DK, Baker CI, Oram MW, Perrett DI. Integration of visual and auditory information by superior temporal sulcus neurons responsive to the sight of actions. J Cogn Neurosci 17: 377-391, 2005.

Barraclough NE, Xiao DK, Oram MW, Perrett DI. The sensitivity of primate STS neurons to walking sequences and to the degree of articulation in static images. Prog Brain Res 154: 135-148, 2006.

Baylis GC, Rolls ET. Responses of neurons in the inferior temporal cortex in short term and serial recognition memory tasks. Exp Brain Res 65: 614622, 1987.

Binzegger T, Douglas RJ, Martin KAC. A quantitative map of the circuit of cat primary visual cortex. J Neurosci 24: 8441-8453, 2004.

Bradley A, Skottun BC, Ohzawa I, Sclar G, Freeman RD. Visual orientation and spatial frequency discrimination: a comparison of single neurons and behavior. J Neurophysiol 57: 755-772, 1987.

Britten KH, Shadlen MN, Newsome WT, Movshon JA. Responses of neurons in macaque MT to stochastic motion signals. Vis Neurosci 10: 1157-1169, 1993.

Bruce C, Desimone R, Gross CG. Visual properties of neurons in a polysensory area in superior temporal sulcus of the macaque. J Neurophysiol 46: 369-384, 1981.

Buracas GT, Zador AM, DeWeese MR, Albright TD. Efficient discrimination of temporal patterns by motion-sensitive neurons in primate visual cortex. Neuron 20: 959-969, 1998.

Carandini M. Amplification of trial-to-trial response variability by neurons in visual cortex. PloS Biol 2: 1483-1493, 2004.

Churchland MM, Yu BM, Ryu SI, Santhanam G, Shenoy KV. Neural variability in premotor cortex provides a signature of motor preparation. $J$ Neurosci 26: 3697-3712, 2006.

Dean AF. The variability of discharge of simple cells in the cat striate cortex. Exp Brain Res 44: 437-440, 1981.

DeAngelis GC, Ghose GM, Ohzawa I, Freeman RD. Functional microorganization of primary visual cortex: receptive field analysis of nearby neurons. J Neurosci 19: 4046-4064, 1999.

de la Rocha J, Doiron B, Shea-Brown E, Josic K, Reyes A. Correlation between neural spike trains increases with firing rate. Nature 448: 802-806, 2007.

de Ruyter van Steveninck R, Lewen GD, Strong SP, Koberle R, Bialek W. Reproducibility and variability in neural spike trains. Science 275: 18051808, 1997.

Douglas RJ, Martin KAC. Recurrent neuronal circuits in the neocortex. Curr Biol 17: R496-R500, 2007.

Dragoi V, Sharma J, Miller EK, Sur M. Dynamics of neuronal sensitivity in visual cortex and local feature discrimination. Nat Neurosci 5: 883-891, 2002.

Edwards R, Xiao DK, Keysers C, Foldiak P, Perrett DI. Color sensitivity of cells responsive to complex stimuli in the temporal cortex. J Neurophysiol 90: 1245-1256, 2003.

Eifuku S, De Souza WC, Tamura R, Nishijo H, Ono T. Neuronal correlates of face identification in the monkey anterior temporal cortical areas. $J$ Neurophysiol 91: 358-371, 2004.

Felleman DJ, Van Essen DC. Distributed hierarchical processing in the primate cerebral cortex. Cereb Cortex 1: 1-47, 1991.

Felsen G, Shen YS, Yao HS, Spor G, Li CY, Dan Y. Dynamic modification of cortical orientation tuning mediated by recurrent connections. Neuron 36: 945-954, 2002.

Foldiak P, Xiao DK, Keysers C, Edwards R, Perrett DI. Rapid serial visual presentation for the determination of neural selectivity in area STSa. Prog Brain Res 144: 107-116, 2003.
Franco L, Rolls ET, Aggelopoulos NC, Treves A. The use of decoding to analyze the contribution to the information of the correlations between the firing of simultaneously recorded neurons. Exp Brain Res 155: 370-384, 2004

Gawne TJ, Kjaer TW, Hertz JA, Richmond BJ. Adjacent visual cortical complex cells share about $20 \%$ of their stimulus-related information. Cereb Cortex 6: 482-489, 1996.

Gawne TJ, Richmond BJ. How independent are the messages carried by adjacent inferior temporal cortical neurons? J Neurosci 13: 2758-2771, 1993.

Geisler WS, Albrecht DG. Visual cortex neurons in monkeys and cats: detection, discrimination, and identification. Vis Neurosci 14: 897-919, 1997.

Gershon ED, Wiener MC, Latham PE, Richmond BJ. Coding strategies in monkey V1 and inferior temporal cortices. J Neurophysiol 79: 1135-1144, 1998.

Grill-Spector K, Henson R, Martin A. Repetition and the brain: neural models of stimulus-specific effects. Trends Cogn Sci 10: 14-23, 2006.

Gur M, Beylin A, Snodderly DM. Response variability of neurons in primary visual cortex (V1) of alert monkeys. J Neurosci 17: 2914-2920, 1997.

Gur M, Snodderly DM. High response reliability of neurons in primary visual cortex (V1) of alert, trained monkeys. Cereb Cortex 16: 888-895, 2006.

Hartveit E, Heggelund P. Response variability of single cells in the dorsal lateral geniculate nucleus of the cat. Comparison with retinal input and effect of brain stem stimulation. J Neurophysiol 72: 1278-1289, 1994.

Kara P, Reinagel P, Reid RC. Low response variability in simultaneously recorded retinal, thalamic, and cortical neurons. Neuron 27: 635-646, 2000.

Kass RE, Ventura V. Spike count correlation increases with length of time interval in the presence of trial-to-trial variation. Neural Comput 18: 25832591, 2006.

Khatri V, Hartings JA, Simons DJ. Adaptation in thalamic barreloid and cortical barrel neurons to periodic whisker deflections varying in frequency and velocity. J Neurophysiol 92: 3244-3254, 2004.

Kiani R, Esteky H, Tanaka K. Differences in onset latency of macaque inferotemporal neural responses to primate and non-primate faces. $\mathrm{J} \mathrm{Neu}$ rophysiol 94: 1587-1596, 2005.

Kohn A, Smith MA. Stimulus dependence of neuronal correlation in primary visual cortex of the macaque. J Neurosci 25: 3661-3673, 2005.

Kourtzi Z, Kanwisher N. Representation of perceived object shape by the human lateral occipital complex. Science 293: 1506-1509, 2001.

Lee D, Port NL, Kruse W, Georgopoulos AP. Variability and correlated noise in the discharge of neurons in motor and parietal areas of the primate cortex. J Neurosci 18: 1161-1170, 1998.

Levine MW, Troy JB. The variability of the maintained discharge of cat dorsal lateral geniculate cells. J Physiol 375: 339-359, 1986.

Macknik SL, Livingstone MS. Neuronal correlates of visibility and invisibility in the primate visual system. Nat Neurosci 1: 144-149, 1998.

Maimon G, Assad JA. Beyond Poisson: increased spike-time regularity across primate parietal cortex. Neuron 62: 426-440, 2009.

Maynard EM, Hatsopoulos NG, Ojakangas CL, Acuna BD, Sanes JN, Normann RA, Donoghue JP. Neuronal interactions improve cortical population coding of movement direction. J Neurosci 19: 8083-8093, 1999.

McAdams CJ, Maunsell JHR. Effects of attention on the reliability of individual neurons in monkey visual cortex. Neuron 23: 765-773, 1999.

Michel MM, Jacobs RA. The costs of ignoring high-order correlations in populations of model neurons. Neural Comput 18: 660-682, 2006.

Muller JR, Metha AB, Krauskopf J, Lennie P. Information conveyed by onset transients in responses of striate cortical neurons. J Neurosci 21: 6978-6990, 2001.

Nirenberg S, Latham PE. Decoding neuronal spike trains: how important are correlations? Proc Natl Acad Sci USA 100: 7348-7353, 2003.

Oram MW. Integrating neuronal coding into cognitive models: predicting reaction time distributions. Network Comput Neural Syst 16: 377-400, 2005

Oram MW, Foldiak P, Perrett DI, Sengpiel F. The "Ideal Homunculus": decoding neural population signals. Trends Neurosci 21: 259-265, 1998.

Oram MW, Hatsopoulos NG, Richmond BJ, Donoghue JP. Excess synchrony in motor cortical neurons provides redundant direction information with that from coarse temporal measures. J Neurophysiol 86: 1700-1716, 2001

Oram MW, Perrett DI. Time course of neural responses discriminating different views of the face and head. J Neurophysiol 68: 70-84, 1992. 
Oram MW, Perrett DI. Integration of form and motion in the anterior superior temporal polysensory area $(\mathrm{STPa})$ of the macaque monkey. $J$ Neurophysiol 76: 109-129, 1996.

Oram MW, Wiener MC, Lestienne R, Richmond BJ. Stochastic nature of precisely timed spike patterns in visual system neuronal responses. $J$ Neurophysiol 81: 3021-3033, 1999.

Oram MW, Xiao DK, Dritschel B, Payne KR. The temporal resolution of neural codes: does response latency have a unique role? Philos Trans $R$ Soc Lond B Biol Sci 357: 987-1001, 2002.

Oram MW, Xiao DK, Endres DM. Stimulus induced decorrelation of neuronal activity in the visual system. Perception 36, ECVP Abstr Suppl: 212, 2007.

Osborne LC, Bialek W, Lisberger SG. Time course of information about motion direction in visual area MT of macaque monkeys. J Neurosci 24: 3210-3222, 2004.

Panzeri S, Schultz SR, Treves A, Rolls ET. Correlations and the encoding of information in the nervous system. Proc $R$ Soc Lond B Biol Sci 266: 1001-1012, 1999.

Perrett DI, Oram MW, Harries MH, Bevan R, Hietanen JK, Benson PJ, Thomas S. Viewer-centred and object-centred coding of heads in the macaque temporal cortex. Exp Brain Res 86: 159-173, 1991.

Perrett DI, Rolls ET, Caan W. Visual neurones responsive to faces in the monkey temporal cortex. Exp Brain Res 47: 329-342, 1982.

Perrett DI, Xiao DK, Barraclough NE, Keysers C, Oram MW. Seeing the future: natural image sequences produce anticipatory neuronal activity and bias perceptual report. Q J Exp Psychol 62: 2081-2104, 2009.

Reich DS, Mechler F, Victor JD. Independent and redundant information in nearby cortical neurons. Science 294: 2566-2568, 2001 a.

Reich DS, Mechler F, Victor JD. Temporal coding of contrast in primary visual cortex: when, what, and why. J Neurophysiol 85: 1039-1050, 2001b.

Reich DS, Victor JD, Knight BW, Ozaki T, Kaplan E. Response variability and timing precision of neuronal spike trains in vivo. $J$ Neurophysiol 77: 2836-2841, 1997.

Reynolds JH, Chelazzi L. Attentional modulation of visual processing. Annu Rev Neurosci 27: 611-647, 2004.

Sary G, Koteles K, Chadaide Z, Tompa T, Benedek G. Task-related modulation in the monkey inferotemporal cortex. Brain Res 1121: 76-82, 2006.

Sawamura H, Orban GA, Vogels R. Selectivity of neuronal adaptation does not match response selectivity: a single-cell study of the fMRI adaptation paradigm. Neuron 49: 307-318, 2006.

Schneidman E, Berry MJ, Segev R, Bialek W. Weak pairwise correlations imply strongly correlated network states in a neural population. Nature 440 : 1007-1012, 2006.

Smith MA, Kohn A. Spatial and temporal scales of neuronal correlation in primary visual cortex. J Neurosci 28: 12591-12603, 2008.

Snedecor GW, Cochran WG. Statistical Methods. Ames, IA: Iowa State Univ. Press, 1980.
Snowden RJ, Treue S, Andersen RA. The response of neurons in areas V1 and MT of the alert rhesus monkey to moving random dot patterns. Exp Brain Res 88: 389-400, 1992.

Stratford KJ, Tarczy-Hornoch K, Martin KA, Bannister NJ, Jack JJ. Excitatory synaptic inputs to spiny stellate cells in cat visual cortex. Nature 382: 258-261, 1996

Teich MC, Turcott RG, Siegel RM. Temporal correlation in cat striate-cortex neural spike trains. IEEE Eng Med Biol Magazine 15: 79-87, 1996.

Tetzlaff T, Rotter S, Stark E, Abeles M, Aertsen A, Diesmann M. Dependence of neuronal correlations on filter characteristics and marginal spike train statistics. Neural Comput 20: 2133-2184, 2008.

Tolhurst DJ, Movshon JA, Dean AF. The statistical reliability of signals in single neurons in cat and monkey visual cortex. Vision Res 23: 775-785, 1983.

Tolhurst DJ, Movshon JA, Thompson ID. The dependence of response amplitude and variance of cat visual cortical neurones on stimulus contrast. Exp Brain Res 41: 414-419, 1981.

Uka T, DeAngelis GC. Contribution of middle temporal area to coarse depth discrimination: comparison of neuronal and psychophysical sensitivity. $J$ Neurosci 23: 3515-3530, 2003.

Van Horn SC, Erisir A, Sherman SM. Relative distribution of synapses in the A-laminae of the lateral geniculate nucleus of the cat. J Comp Neurol 416: 509-520, 2000.

van Rossum MCW, van der Meer M, Xiao D, Oram MW. Adaptive integration by recurrent cortical circuits. Neural Comput 20: 1847-1872, 2008.

Victor JD, Purpura KP. Nature and precision of temporal coding in visual cortex: a metric-space analysis. J Neurophysiol 76: 1310-1326, 1996.

Vogels R, Orban GA. Quantitative study of striate single unit responses in monkeys performing an orientation discrimination task. Exp Brain Res 84: 1-11, 1991.

Vogels R, Spileers W, Orban GA. The response variability of striate cortical neurons in the behaving monkey. Exp Brain Res 77: 432-436, 1989.

Wehr M, Zador AM. Synaptic mechanisms of forward suppression in rat auditory cortex. Neuron 47: 437-445, 2005.

Wiener MC, Oram MW, Liu Z, Richmond BJ. Consistency of encoding in monkey visual cortex. J Neurosci 21: 8210-8221, 2001.

Wilke SD, Eurich CW. Neural spike statistics modify the impact of background noise. Neurocomputing 38-40: 445-450, 2001.

Wilke SD, Eurich CW. On the functional role of noise correlations in the nervous system. Neurocomputing 44-46: 1023-1028, 2002.

Young MP. The organization of neural systems in the primate cerebral cortex. Proc R Soc Lond B Biol Sci 252: 13-18, 1993.

Young MP, Scannell JW, Burns GA, Blakemore C. Analysis of connectivity: neural systems in the cerebral cortex. Rev Neurosci 5: 227-250, 1994.

Zohary E, Shadlen MN, Newsome WT. Correlated neuronal discharge rate and its implications for psychophysical performance. Nature 370: 140-143, 1994. 